\title{
Active Constrained Layer Damping of Smart Skew Laminated Composite Plates Using 1-3 Piezoelectric Composites
}

\author{
R. M. Kanasogi and M. C. Ray \\ Department of Mechanical Engineering, Indian Institute of Technology, Kharagpur 721302, India \\ Correspondence should be addressed to M. C. Ray; mcray@mech.iitkgp.ernet.in
}

Received 20 January 2013; Accepted 5 April 2013

Academic Editor: Hui Shen Shen

Copyright (c) 2013 R. M. Kanasogi and M. C. Ray. This is an open access article distributed under the Creative Commons Attribution License, which permits unrestricted use, distribution, and reproduction in any medium, provided the original work is properly cited.

\begin{abstract}
This paper deals with the analysis of active constrained layer damping (ACLD) of smart skew laminated composite plates. The constraining layer of the ACLD treatment is composed of the vertically/obliquely reinforced 1-3 piezoelectric composites (PZCs). A finite element model has been developed for accomplishing the task of the active constrained layer damping of skew laminated symmetric and antisymmetric cross-ply and antisymmetric angle-ply composite plates integrated with the patches of such ACLD treatment. Both in-plane and out-of-plane actuations by the constraining layer of the ACLD treatment have been utilized for deriving the finite element model. The analysis revealed that the vertical actuation dominates over the in-plane actuation. Particular emphasis has been placed on investigating the performance of the patches when the orientation angle of the piezoelectric fibers of the constraining layer is varied in the two mutually orthogonal vertical planes. Also, the effects of varying the skew angle of the substrate laminated composite plates and different boundary conditions on the performance of the patches have been studied. The analysis reveals that the vertically and the obliquely reinforced 1-3 PZC materials should be used for achieving the best control authority of ACLD treatment, as the boundary conditions of the smart skew laminated composite plates are simply supported and clamped-clamped, respectively.
\end{abstract}

\section{Introduction}

Extensive research on the use of piezoelectric materials for making distributed actuators and sensors of light weight flexible smart structures has been carried out during the past several years [1-16]. The distributed piezoelectric actuators and sensors are either mounted on or embedded into the host flexible light weight structures. When they are activated with proper control voltage, the resulting structures attain self-controlling and self-sensing capabilities. Such flexible structures having built-in mechanism for self-controlling and self-sensing capabilities are customarily called "smart structures." In most of the work on smart structures, the distributed actuators were considered to be made of the existing monolithic piezoelectric materials. The magnitudes of the piezoelectric coefficients of the existing monolithic piezoelectric materials are very small. Hence, the distributed actuators made of these materials need large control voltage for satisfactory control of smart structures. The further research on the efficient use of these low-control authority monolithic piezoelectric materials led to the development of the active constrained layer damping (ACLD) treatment [17]. The ACLD treatment consists of a constraining layer made of the piezoelectric materials and a constrained viscoelastic layer. The flexural vibration control by the constrained layer damping treatment is attributed to the dissipation of energy in the constrained viscoelastic layer due to its transverse shear deformations. The constraining layer of the activated ACLD treatment increases the transverse shear deformations of the viscoelastic constrained layer over its passive counterpart resulting in improved damping of the host structures. The control voltage required to cause transverse shear deformations in the low-stiff constrained viscoelastic layer of the ACLD treatment is compatible with the low-control authority of the monolithic piezoelectric materials. Hence, the piezoelectric materials perform much better to attenuate the vibrations of smart structures when they are used for the constraining layer of the ACLD treatment than when 
they are directly bonded to the flexible host structures. If the constraining piezoelectric layer of the ACLD treatment is not activated with the control voltage, the treatment causes the standard passive constrained layer damping of the smart structure. Thus, the ACLD treatment being under operation provides the attributes of both passive and active dampings and acts as an inbuilt fail-safe mechanism. Since its inception, extensive research has been carried out to investigate the performance of the ACLD treatment for active damping of smart structures [18-25].

Piezoelectric composite (PZC) materials have been emerged as the new class of smart materials. Such PZC materials are composed of piezoelectric fiber reinforcements and epoxy matrix. These PZC materials provide wide range of effective material properties, good conformability, and strength integrity [26]. Among the various PZC materials studied by the researchers, the vertically and the obliquely reinforced 1-3 PZC materials are commercially available [27] and are being effectively used for underwater transducers, medical imaging applications, and high frequency ultrasonic transducers [26]. The constructional feature of a lamina made of the vertically reinforced 1-3 PZC material is that the piezoelectric fibers are vertically aligned across the thickness of the lamina. In case of the obliquely reinforced 1-3 PZC, the piezoelectric fibers are obliquely aligned in the vertical plane across the thickness of the lamina. These PZCs are characterized by improved mechanical performance, electromechanical coupling characteristics, and acoustic impedance matching over the existing monolithic piezoelectric materials [26]. Research on PZC materials is mainly concerned with the micromechanical analysis of these materials [28-34]. Recently, Ray and his coworkers [35-37] investigated the performance of these 1-3 PZC materials for active damping of linear and nonlinear vibrations of composite beams, plates and shells.

Skew laminated composite plates are widely used in engineering applications such as aircraft wings and marine industries and are highly prone to undergoing vibrations. Researchers extensively investigated the free vibrational characteristics of such skew plates. For example, Krishnan and Deshpande [38] carried out the free vibration analyses of cantilevered skew isotropic platesand three-layered symmetric cross-ply laminates by deriving two finite element models. Krishna Reddy and Palaninathan [39] developed a general high precision triangular plate bending finite element for the free vibration analysis of laminated skew plates. Babu and Kant [40] developed shear deformable finite element models for the buckling analysis of skew composite plates and panels. Garg et al. [41] developed a simple $\mathrm{C}^{\circ}$ isoparametric finite element model, based on a higher order shear deformation theory for the free vibration analysis of isotropic, orthotropic, and layered anisotropic composite and sandwich skew laminates.

The review of the existing literature on the skew plates reveals that the attention has not yet been focused on investigating the active control of vibrations of skew laminated composite plates using piezoelectric composites. In this paper, authors intend to investigate the active constrained layer damping (ACLD) of skew laminated composite plates. For such investigation, three-dimensional analysis of ACLD of skew laminated composite plates integrated with the patches of ACLD treatment has been carried out by the finite element method. The constraining layer of the ACLD treatment is considered to be made of the vertically or the obliquely reinforced 1-3 PZC materials. Particular emphasis has been placed on investigating the effect of variation of piezoelectric fiber orientation angle on the performance of the ACLD patches for controlling the vibrations of the skew laminated composite plates.

\section{Finite Element Model of Smart Skew Laminated Composite Plate}

Figure 1(a) illustrates the schematic diagram of a smart skew laminated composite plate composed of $N$ number of orthotropic layers. The length and the skew width of the plate are denoted by $a$ and $b$, respectively. The top surface of the plate is integrated with the skewed patches of the ACLD treatment. The constraining layer of the ACLD treatment is made of the vertically/obliquely reinforced 13 piezoelectric composite (PZC) material. The notations $h$, $h_{p}$, and $h_{v}$ represent the thicknesses of the substrate skew laminated plate, the piezoelectric composite layer, and the viscoelastic layer, respectively. The skew angle of the plate is denoted by $\alpha$. The middle plane of the substrate composite plate is considered as the reference plane. The origin of the laminate coordinate system $(x y z)$ is located at one corner of the reference plane such that the lines $y=0$ and $y=$ $b \cos \alpha$ represent the two opposite boundaries of the substrate skew composite plates, while the lines $x=y \tan \alpha$ and $x=a+y \tan \alpha$ describe the two opposite skewed edges of the plate. Denoting by $k(=1,2,3, \ldots, N+2)$ the layer number of any layer of the overall plate, the thickness coordinates $z$ of the top and the bottom surfaces of any ( $k$ th) layer are represented by $h_{k+1}$ and $h_{k}$, respectively. The fiber orientation angle in any layer of the substrate plate in the plane $(x y)$ of the lamina with respect to the laminate coordinate system is denoted by $\theta$. The piezoelectric fibers in the constraining layer made of the obliquely reinforced 1-3 PZC material are coplanar with the vertical $x z$ - or $y z$-plane making an angle $\psi$ with respect to the $z$-axis as shown in Figure 1(b). If the value of $\psi$ is $0^{\circ}$, the layer becomes vertically reinforced 1-3 PZC layer. The overall skew composite plate being studied here is thin, and consequently, the first order shear deformation theory (FSDT) can be used to model the axial displacements in all the layers of the overall plate. In Figure 2, the kinematics of axial deformations of the overall plate based on the FSDT has been illustrated. Displayed in this figure, the variables $u_{0}$ and $v_{0}$ represent the generalized translational displacement of a point $(x, y)$ on the reference plane $(z=0)$ along $x$ - and $y$-directions, respectively; $\theta_{x}$, $\phi_{x}$, and $\gamma_{x}$ denote the generalized rotations of the normal to the middle planes of the substrate plate, the viscoelastic layer and the 1-3 PZC layer, respectively, about the $y$-axis, while $\theta_{y}, \phi_{y}$ and $\gamma_{y}$ represent the generalized rotations of the same about the $x$-axis, respectively. According to the kinematics of deformations illustrated in Figure 2, the axial displacements 


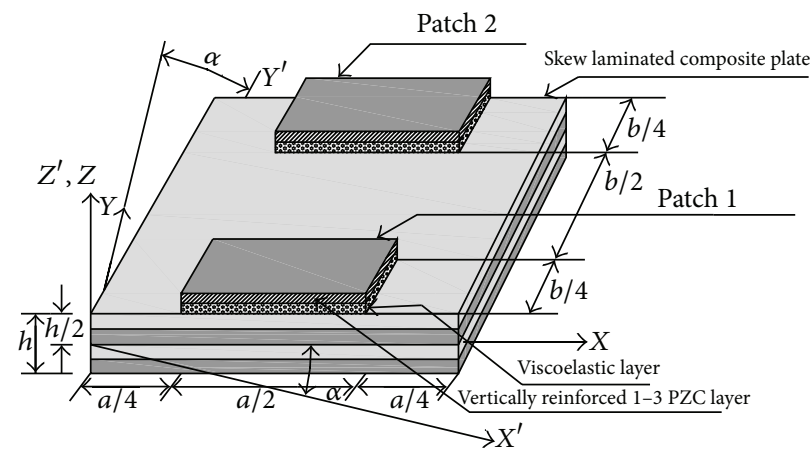

(a)

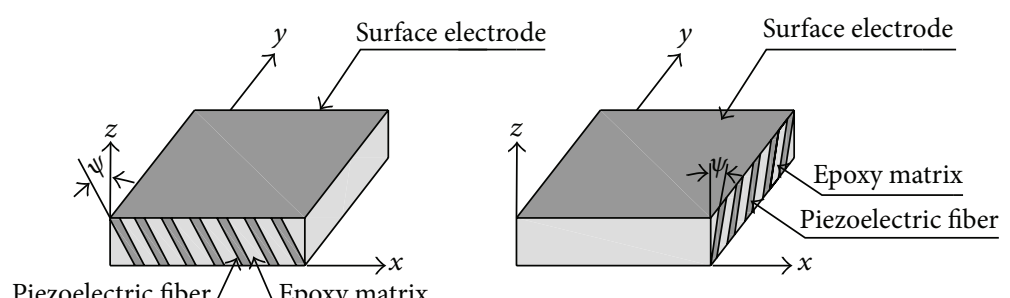

(b)

FIgURE 1: (a) Schematic representation of a skew laminated composite plate integrated with the patches of ACLD treatment composed of vertically/obliquely reinforced 1-3 piezoelectric composite constraining layer. (b) Schematic diagram of layers of obliquely reinforced 1-3 piezoelectric composite.
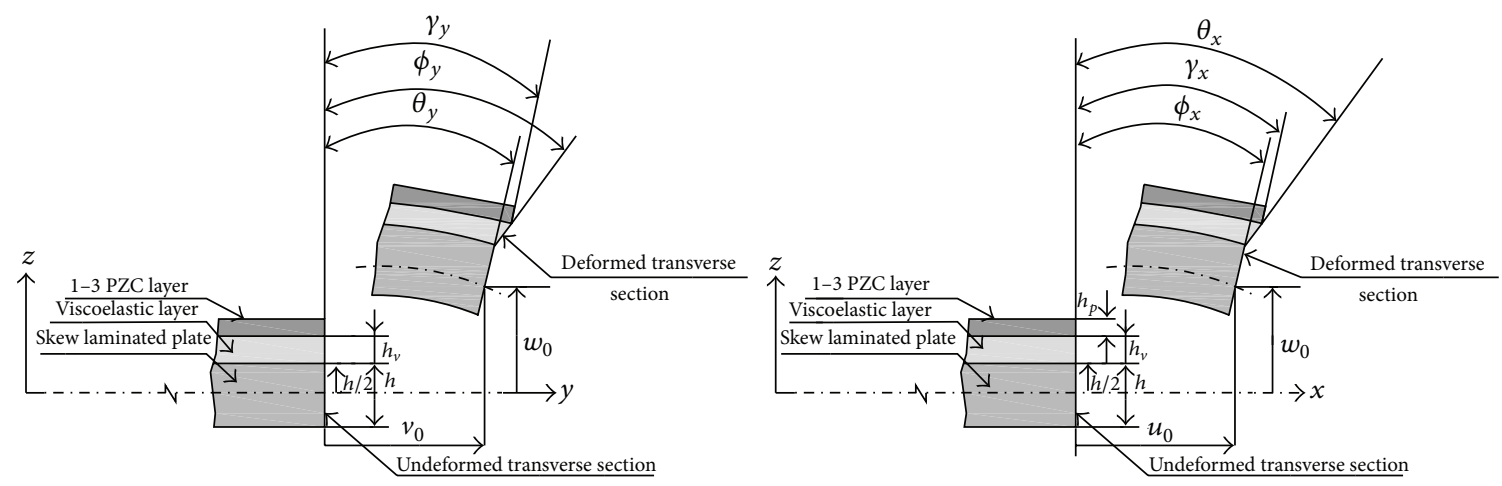

FIGURE 2: Kinematics of deformations.

$u$ and $v$ at any point in any layer of the overall plate along $x$ and $y$-directions, respectively, can be expressed as

$$
\begin{aligned}
u(x, y, z, t)= & u_{0}(x, y, t)+\left(z-\left\langle z-\frac{h}{2}\right\rangle\right) \theta_{x}(x, y, t) \\
& +\left(\left\langle z-\frac{h}{2}\right\rangle-\left\langle z-h_{N+2}\right\rangle\right) \phi_{x}(x, y, t) \\
& +\left\langle z-h_{N+2}\right\rangle \gamma_{x}(x, y, t), \\
v(x, y, z, t) & =v_{0}(x, y, t)+\left(z-\left\langle z-\frac{h}{2}\right\rangle\right) \theta_{y}(x, y, t)
\end{aligned}
$$

$$
\begin{aligned}
& +\left(\left\langle z-\frac{h}{2}\right\rangle-\left\langle z-h_{N+2}\right\rangle\right) \phi_{y}(x, y, t) \\
& +\left\langle z-h_{N+2}\right\rangle \gamma_{y}(x, y, t),
\end{aligned}
$$

in which a function within the bracket $\langle\cdot\rangle$ represents an appropriate singularity function, which satisfies the continuity of displacements between two adjacent continua. Since the transverse actuation of the constraining layer of the ACLD treatment will be used for the flexural vibration control of the plate, the transverse normal strain in the overall plate must be considered in the model. Hence, as the plate considered here is thin, the transverse displacements $(w)$ at any point in the substrate plate, the viscoelastic layer, and 
the 1-3 PZC layer are assumed to be linearly varying across their thicknesses. Thus, similar to the axial displacement, the transverse displacements at any point in the overall plate can be expressed as

$$
\begin{aligned}
w(x, y, z, t)= & w_{0}(x, y, t)+\left(z-\left\langle z-\frac{h}{2}\right\rangle\right) \theta_{z}(x, y, t) \\
& +\left(\left\langle z-\frac{h}{2}\right\rangle-\left\langle z-h_{N+2}\right\rangle\right) \phi_{z}(x, y, t) \\
& +\left\langle z-h_{N+2}\right\rangle \gamma_{z}(x, y, t),
\end{aligned}
$$

in which $w_{0}$ refers to the transverse displacement at any point on the reference plane, and $\theta_{z}, \phi_{z}$, and $\gamma_{z}$ are the generalized displacements representing the gradients of the transverse displacement in the substrate plate, the viscoelastic layer, and the 1-3 PZC layer, respectively, with respect to the thickness coordinate $(z)$.

For the ease of analysis, the generalized displacement variables are grouped into the following two vectors:

$$
\begin{aligned}
& \left\{d_{t}\right\}=\left[\begin{array}{lll}
u_{0} & v_{0} & w_{0}
\end{array}\right]^{T}, \\
& \left\{d_{r}\right\}=\left[\begin{array}{lllllllll}
\theta_{x} & \theta_{y} & \theta_{z} & \phi_{x} & \phi_{y} & \phi_{z} & \gamma_{x} & \gamma_{y} & \gamma_{z}
\end{array}\right]^{T} \text {. }
\end{aligned}
$$

In order to implement the selective integration rule for computing the element stiffness matrices corresponding to the transverse shear deformations, the state of strain at any point in the overall plate is divided into the following two strain vectors $\left\{\varepsilon_{b}\right\}$ and $\left\{\varepsilon_{s}\right\}$ :

$$
\left\{\varepsilon_{b}\right\}=\left[\begin{array}{llll}
\varepsilon_{x} & \varepsilon_{y} & \varepsilon_{x y} & \varepsilon_{z}
\end{array}\right]^{T}, \quad\left\{\varepsilon_{s}\right\}=\left[\begin{array}{ll}
\varepsilon_{x z} & \varepsilon_{y z}
\end{array}\right]^{T}
$$

in which $\varepsilon_{x}, \varepsilon_{y}$, and $\varepsilon_{z}$ are the normal strains along $x$-, $y$-, and $z$-directions, respectively; $\varepsilon_{x y}$ is the in-plane shear strain; $\varepsilon_{x z}$ and $\varepsilon_{y z}$ are the transverse shear strains. Using the displacement fields given by (1)-(3) and the linear straindisplacement relations, the vectors $\left\{\varepsilon_{b}\right\}_{c},\left\{\varepsilon_{b}\right\}_{v}$, and $\left\{\varepsilon_{b}\right\}_{p}$ defining the state of in-plane and transverse normal strains at any point in the substrate skew composite plate, the viscoelastic layer, and the active constraining layer, respectively, can be expressed as

$$
\begin{gathered}
\left\{\varepsilon_{b}\right\}_{c}=\left\{\varepsilon_{b t}\right\}+\left[Z_{1}\right]\left\{\varepsilon_{b r}\right\}, \quad\left\{\varepsilon_{b}\right\}_{v}=\left\{\varepsilon_{b t}\right\}+\left[Z_{2}\right]\left\{\varepsilon_{b r}\right\} \\
\left\{\varepsilon_{b}\right\}_{p}=\left\{\varepsilon_{b t}\right\}+\left[Z_{3}\right]\left\{\varepsilon_{b r}\right\} .
\end{gathered}
$$

Similarly, the vectors $\left\{\varepsilon_{s}\right\}_{c},\left\{\varepsilon_{s}\right\}_{v}$, and $\left\{\varepsilon_{s}\right\}_{p}$ defining the state of transverse shear strains at any point in the substrate composite plate, the viscoelastic layer, and the active constraining layer, respectively, can be expressed as

$$
\begin{gathered}
\left\{\varepsilon_{s}\right\}_{c}=\left\{\varepsilon_{s t}\right\}+\left[Z_{4}\right]\left\{\varepsilon_{s r}\right\}, \quad\left\{\varepsilon_{s}\right\}_{v}=\left\{\varepsilon_{s t}\right\}+\left[Z_{5}\right]\left\{\varepsilon_{s r}\right\}, \\
\left\{\varepsilon_{s}\right\}_{p}=\left\{\varepsilon_{s t}\right\}+\left[Z_{6}\right]\left\{\varepsilon_{s r}\right\} .
\end{gathered}
$$

The various matrices appearing in (6) and (7) have been defined in the Appendix while the generalized strain vectors are given by

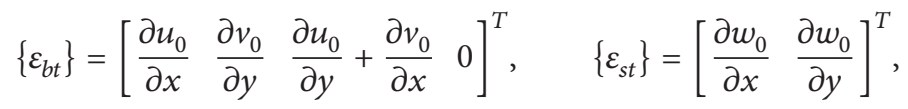

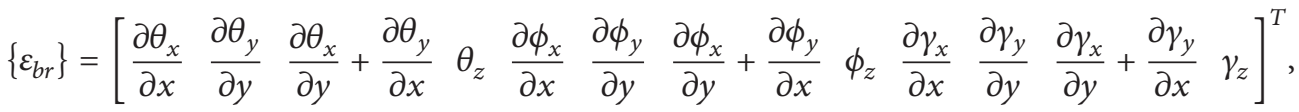

$$
\begin{aligned}
& \left\{\varepsilon_{s r}\right\}=\left[\begin{array}{llllllllllll}
\theta_{x} & \theta_{y} & \phi_{x} & \phi_{y} & \gamma_{x} & \gamma_{y} & \frac{\partial \theta_{z}}{\partial x} & \frac{\partial \theta_{z}}{\partial y} & \frac{\partial \phi_{z}}{\partial x} & \frac{\partial \phi_{z}}{\partial y} & \frac{\partial \gamma_{z}}{\partial x} & \frac{\partial \gamma_{z}}{\partial y}
\end{array}\right]^{T}
\end{aligned}
$$

Similar to the strain vectors given by (5), the state of stresses at any point in the overall plat is described by the following stress vectors:

$$
\left\{\sigma_{b}\right\}=\left[\begin{array}{llll}
\sigma_{x} & \sigma_{y} & \sigma_{x y} & \sigma_{z}
\end{array}\right]^{T}, \quad\left\{\sigma_{s}\right\}=\left[\begin{array}{ll}
\sigma_{x z} & \sigma_{y z}
\end{array}\right]^{T},
$$

where $\sigma_{x}, \sigma_{y}$, and $\sigma_{z}$ are the normal stresses along $x$-, $y$ and $z$-directions, respectively; $\sigma_{x y}$ is the in-plane shear stress; $\sigma_{x z}$ and $\sigma_{y z}$ are the transverse shear stresses. The constitutive relations for the material of any orthotropic layer of the substrate plate are given by

$$
\begin{array}{r}
\left\{\sigma_{b}^{k}\right\}=\left[C_{b}^{k}\right]\left\{\varepsilon_{b}^{k}\right\}, \quad\left\{\sigma_{s}^{k}\right\}=\left[C_{s}^{k}\right]\left\{\varepsilon_{s}^{k}\right\} \\
(k=1,2,3, \ldots, N),
\end{array}
$$


where the elastic coefficient matrices are

$$
\begin{aligned}
& {\left[C_{b}^{k}\right]=\left[\begin{array}{llll}
\bar{C}_{11}^{k} & \bar{C}_{12}^{k} & \bar{C}_{16}^{k} & \bar{C}_{13}^{k} \\
\bar{C}_{12}^{k} & \bar{C}_{22}^{k} & \bar{C}_{26}^{k} & \bar{C}_{23}^{k} \\
\bar{C}_{16}^{k} & \bar{C}_{26}^{k} & \bar{C}_{66}^{k} & \bar{C}_{36}^{k} \\
\bar{C}_{13}^{k} & \bar{C}_{23}^{k} & \bar{C}_{36}^{k} & \bar{C}_{33}^{k}
\end{array}\right],} \\
& {\left[C_{s}^{k}\right]=\left[\begin{array}{ll}
\bar{C}_{55}^{k} & \bar{C}_{45}^{k} \\
\bar{C}_{45}^{k} & \bar{C}_{44}^{k}
\end{array}\right],}
\end{aligned}
$$

and $\bar{C}_{i j}^{k}(i, j=1,2,3, \ldots, 6)$ are the transformed elastic coefficients with respect to the reference coordinate system. The material of the viscoelastic layer is assumed to be linearly viscoelastic and homogenous isotropic and is modeled by using the complex modulus approach. Thus, the shear modulus $G$ and Young's modulus $E$ of the viscoelastic material are given by

$$
G=G^{\prime}(1+i \eta), \quad E=2 G(1+\nu),
$$

in which $G^{\prime}$ is the storage modulus, $v$ is Poisson's ratio, and $\eta$ is the loss factor at a particular operating temperature and frequency. Employing the complex modulus approach, the constitutive relations for the material of the viscoelastic layer $(k=N+1)$ can also be represented by (10) with $\bar{C}_{i j}^{k}(i, j=$ $1,2,3, \ldots, 6)$ being the complex elastic constants $[29,30]$. The constraining PZC layer will be subjected to the applied electric field $\left(E_{z}\right)$ acting across its thickness (i.e., along the $z$-direction) only. Accordingly, the constitutive relations for the 1-3 PZC material with respect to the laminate coordinate system $(x y z)$ can be expressed as

$$
\begin{array}{r}
\left\{\sigma_{b}^{k}\right\}=\left[\bar{C}_{b}^{k}\right]\left\{\varepsilon_{b}^{k}\right\}+\left[\bar{C}_{b s}^{k}\right]\left\{\varepsilon_{s}^{k}\right\}-\left\{e_{b}\right\} E_{z}, \\
\left\{\sigma_{s}^{k}\right\}=\left[\bar{C}_{b s}^{k}\right]\left\{\varepsilon_{b}^{k}\right\}+\left[\bar{C}_{s}^{k}\right]\left\{\varepsilon_{s}^{k}\right\}-\left\{e_{s}\right\} E_{z} \\
D_{z}=\left\{e_{b}\right\}^{T}\left\{\varepsilon_{b}^{k}\right\}+\left\{e_{s}\right\}^{T}\left\{\varepsilon_{s}^{k}\right\}+\bar{\varepsilon}_{33} E_{z} \\
k=N+2 .
\end{array}
$$

Here, $D_{z}$ represents the electric displacement along the $z$ direction and $\bar{\varepsilon}_{33}$ is the dielectric constant. It may be noted from the previous form of the constitutive relations that the transverse shear strains are coupled with the in-plane normal strains due to the orientation of piezoelectric fibers in the vertical $x z$ - or $y z$-plane, and the corresponding coupling elastic constant matrices $\left[\bar{C}_{b s}^{N+2}\right]$ are given by

$$
\left[\bar{C}_{b s}^{N+2}\right]=\left[\begin{array}{cc}
\bar{C}_{15}^{N+2} & 0 \\
\bar{C}_{25}^{N+2} & 0 \\
0 & \bar{C}_{46}^{N+2} \\
\bar{C}_{35}^{N+2} & 0
\end{array}\right]
$$

$$
\left[\bar{C}_{b s}^{N+2}\right]=\left[\begin{array}{cc}
0 & \bar{C}_{14}^{N+2} \\
0 & \bar{C}_{24}^{N+2} \\
\bar{C}_{56}^{N+2} & 0 \\
0 & \bar{C}_{34}^{N+2}
\end{array}\right]
$$

as the piezoelectric fibers are coplanar with the vertical $x z$ or $y z$-plane, respectively. Note that if the fibers are coplanar with both $x z$ - and $y z$-planes (i.e., $\psi=0^{\circ}$ ), this coupling matrix becomes a null matrix. Also, the piezoelectric constant matrices $\left\{e_{b}\right\}$ and $\left\{e_{s}\right\}$ appearing in (13) contain the following transformed effective piezoelectric coefficients of the 1-3 PZC:

$$
\left\{e_{b}\right\}=\left[\begin{array}{llll}
\bar{e}_{31} & \bar{e}_{32} & \bar{e}_{36} & \bar{e}_{33}
\end{array}\right]^{T}, \quad\left\{e_{s}\right\}=\left[\begin{array}{ll}
\bar{e}_{35} & \bar{e}_{34}
\end{array}\right]^{T} .
$$

The total potential energy $T_{p}$ and the kinetic energy $T_{k}$ of the overall plate/ACLD system are given by [35]

$$
\begin{gathered}
T_{p}=\frac{1}{2}\left[\sum_{k=1}^{N+2} \int_{\Omega}\left(\left\{\varepsilon_{b}^{k}\right\}^{T}\left\{\sigma_{b}^{k}\right\}+\left\{\varepsilon_{s}^{r}\right\}^{T}\left\{\sigma_{s}^{k}\right\}\right) d \Omega\right. \\
\left.-\int_{\Omega} D_{z} E_{z} d \Omega\right]-\int_{A}\{d\}^{T}\{f\} d A, \\
T_{k}=\frac{1}{2} \sum_{k=1}^{N+2} \int_{\Omega} \rho^{k}\left(\dot{u}^{2}+\dot{v}^{2}+\dot{w}^{2}\right) d \Omega,
\end{gathered}
$$

in which $\rho^{k}$ is the mass density of the $k$ th layer, $\{f\}$ is the externally applied surface traction acting over a surface area $A$, and $\Omega$ represents the volume of the concerned layer. Since the plates under study are considered to be thin, the rotary inertia of the overall plate has been neglected in estimating the kinetic energy. The overall plate is discretized by eightnoded isoparametric quadrilateral elements. Following (4), the generalized displacement vectors, associated with the $i$ th $(i=1,2,3, \ldots, 8)$ node of the element, can be written as

$$
\begin{aligned}
& \left\{d_{t i}\right\}=\left[\begin{array}{lll}
u_{0 i} & v_{0 i} & w_{0 i}
\end{array}\right]^{T} \\
& \left\{d_{r i}\right\}=\left[\begin{array}{lllllllll}
\theta_{x i} & \theta_{y i} & \theta_{z i} & \phi_{x i} & \phi_{y i} & \phi_{z i} & \gamma_{x i} & \gamma_{y i} & \gamma_{z i}
\end{array}\right]^{T} \text {. }
\end{aligned}
$$


Thus, the generalized displacement vectors at any point within the element can be expressed in terms of the nodal generalized displacement vectors $\left\{d_{t}^{e}\right\}$ and $\left\{d_{r}^{e}\right\}$ as follows:

$$
\left\{d_{t}^{e}\right\}=\left[N_{t}\right]\left\{d_{t}^{e}\right\}, \quad\left\{d_{r}^{e}\right\}=\left[N_{r}\right]\left\{d_{r}^{e}\right\},
$$

in which

$$
\begin{gathered}
\left\{d_{t}^{e}\right\}=\left[\begin{array}{llll}
\left\{d_{t 1}^{e}\right\}^{T} & \left\{d_{t 2}^{e}\right\}^{T} & \cdots & \left\{d_{t 8}^{e}\right\}^{T}
\end{array}\right]^{T}, \\
\left\{d_{r}^{e}\right\}=\left[\begin{array}{llll}
\left\{d_{r 1}^{e}\right\}^{T} & \left\{d_{r 2}^{e}\right\}^{T} & \cdots & \left\{d_{r 8}^{e}\right\}^{T}
\end{array}\right]^{T}, \\
{\left[N_{t}\right]=\left[\begin{array}{llll}
N_{t 1} & N_{t 2} & \cdots & N_{t 8}
\end{array}\right]^{T},} \\
{\left[\begin{array}{llll}
N_{r}
\end{array}\right]=\left[\begin{array}{llll}
N_{r 1} & N_{r 2} & \cdots & N_{r 8}
\end{array}\right]^{T},} \\
N_{t i}=n_{i} I_{t}, \quad N_{r i}=n_{i} I_{r},
\end{gathered}
$$

while $I_{t}$ and $I_{r}$ are $(3 \times 3)$ and $(9 \times 9)$ identity matrices, respectively, and $n_{i}$ is the shape function of natural coordinates associated with the $i$ th node. Making use of the relations given by (6)-(8) and (18), the strain vectors at any point within the element can be expressed in terms of the nodal generalized displacement vectors as follows:

$$
\begin{aligned}
& \left\{\varepsilon_{b}\right\}_{c}=\left[B_{t b}\right]\left\{d_{t}^{e}\right\}+\left[Z_{1}\right]\left[B_{r b}\right]\left\{d_{r}^{e}\right\}, \\
& \left\{\varepsilon_{b}\right\}_{v}=\left[B_{t b}\right]\left\{d_{t}^{e}\right\}+\left[Z_{2}\right]\left[B_{r b}\right]\left\{d_{r}^{e}\right\}, \\
& \left\{\varepsilon_{b}\right\}_{p}=\left[B_{t b}\right]\left\{d_{t}^{e}\right\}+\left[Z_{3}\right]\left[B_{r b}\right]\left\{d_{r}^{e}\right\}, \\
& \left\{\varepsilon_{s}\right\}_{c}=\left[B_{t s}\right]\left\{d_{t}^{e}\right\}+\left[Z_{4}\right]\left[B_{r s}\right]\left\{d_{r}^{e}\right\}, \\
& \left\{\varepsilon_{s}\right\}_{v}=\left[B_{t s}\right]\left\{d_{t}^{e}\right\}+\left[Z_{5}\right]\left[B_{r s}\right]\left\{d_{r}^{e}\right\}, \\
& \left\{\varepsilon_{s}\right\}_{p}=\left[B_{t s}\right]\left\{d_{t}^{e}\right\}+\left[Z_{6}\right]\left[B_{r s}\right]\left\{d_{r}^{e}\right\},
\end{aligned}
$$

in which the nodal strain-displacement matrices $\left[B_{t b}\right],\left[B_{r b}\right]$, $\left[B_{t s}\right]$, and $\left[B_{r s}\right]$ are given by

$$
\begin{aligned}
& {\left[B_{t b}\right]=\left[\begin{array}{llll}
B_{t b 1} & B_{t b 2} & \cdots & B_{t b 8}
\end{array}\right],} \\
& {\left[B_{r b}\right]=\left[\begin{array}{llll}
B_{r b 1} & B_{r b 2} & \cdots & B_{r b 8}
\end{array}\right],} \\
& {\left[B_{t s}\right]=\left[\begin{array}{llll}
B_{t s 1} & B_{t s 2} & \cdots & B_{t s 8}
\end{array}\right],} \\
& {\left[B_{r s}\right]=\left[\begin{array}{llll}
B_{r s 1} & B_{r s 2} & \cdots & B_{r s 8}
\end{array}\right] .}
\end{aligned}
$$

The submatrices of $\left[B_{t b}\right],\left[B_{r b}\right],\left[B_{t s}\right]$, and $\left[B_{r s}\right]$ have been explicitly presented in the Appendix. On substitution of (9)(13) and (20) into (16), the total potential energy $T_{p}^{e}$ and the kinetic energy $T_{k}^{e}$ of a typical element augmented with the ACLD treatment can be expressed as

$$
\begin{gathered}
T_{p}^{e}=\frac{1}{2}\left[\left\{d_{t}^{e}\right\}^{T}\left[K_{t t}^{e}\right]\left\{d_{t}^{e}\right\}+\left\{d_{t}^{e}\right\}^{T}\left[K_{t r}^{e}\right]\left\{d_{r}^{e}\right\}+\left\{d_{r}^{e}\right\}^{T}\left[K_{t r}^{e}\right]^{T}\right. \\
\times\left\{d_{t}^{e}\right\}+\left\{d_{r}^{e}\right\}^{T}\left[K_{r r}^{e}\right]\left\{d_{r}^{e}\right\}-2\left\{d_{t}^{e}\right\}^{T}\left\{F_{t p}^{e}\right\} V \\
\left.-2\left\{d_{r}^{e}\right\}^{T}\left\{F_{r p}^{e}\right\} V-2\left\{d_{t}^{e}\right\}^{T}\left\{F^{e}\right\}-\varepsilon_{33} \frac{V^{2}}{h_{p}}\right] \\
T_{k}^{e}=\frac{1}{2}\left\{\dot{d}_{t}^{e}\right\}^{T}\left[M^{e}\right]\left\{\dot{d}_{t}^{e}\right\}
\end{gathered}
$$

In (22), $V$ represents the voltage difference applied across the thickness of the PZC layer. The elemental stiffness matrices $\left[K_{t t}^{e}\right],\left[K_{t r}^{e}\right]$ and $\left[K_{r r}^{e}\right]$, the elemental electroelastic coupling vectors $\left\{F_{t p}^{e}\right\}$ and $\left\{F_{r p}^{e}\right\}$, the elemental load vector $\left\{F^{e}\right\}$, and the elemental mass matrix $\left[M^{e}\right]$ appearing in (22) and (23) are given by

$$
\begin{gathered}
{\left[K_{t t}^{e}\right]=\left[K_{t b}^{e}\right]+\left[K_{t s}^{e}\right]+\left[K_{t b s}^{e}\right]_{p b}+\left[K_{t b s}^{e}\right]_{p s},} \\
{\left[K_{t r}^{e}\right]=\left[K_{t r b}^{e}\right]+\left[K_{t r s}^{e}\right]} \\
+\frac{1}{2}\left(\left[K_{t r b s}^{e}\right]_{p b}+\left[K_{r t b s}^{e}\right]_{p b}^{T}+\left[K_{t r b s}^{e}\right]_{p s}+\left[K_{r t b s}^{e}\right]_{p s}^{T}\right), \\
{\left[K_{r t}^{e}\right]=\left[K_{t r}^{e}\right]^{T},} \\
\left\{K_{r r}^{e}\right]=\left[K_{r r b}^{e}\right]+\left[K_{r r s}^{e}\right]+\left[K_{r r b s}^{e}\right]_{p b}+\left[K_{r r b s}^{e}\right]_{p s}, \\
\left\{F_{t p}^{e}\right\}=\left\{F_{t b}^{e}\right\}_{p}+\left\{F_{t s}^{e}\right\}_{p}, \quad\left\{F_{r p}^{e}\right\}=\left\{F_{r b}^{e}\right\}_{p}+\left\{F_{r s}^{e}\right\}_{p}, \\
\left\{F^{e}\right\}=\int_{0}^{a_{e}} \int_{0}^{b_{e}}\left[N_{t}\right]^{T}\{f\} d x d y, \\
\int_{0}^{a_{e}} \int_{0}^{b_{e}} \bar{m}[N]^{T}[N] d x d y, \quad \bar{m}=\sum_{k=1}^{N+2} \rho^{k}\left(h_{k+1}-h_{k}\right),
\end{gathered}
$$

where

$$
\begin{gathered}
{\left[K_{t b}^{e}\right]=\int_{A}\left[B_{t b}\right]^{T}\left(\left[D_{t b}\right]+\left[D_{t b}\right]_{v}+\left[D_{t b}\right]_{p}\right)\left[B_{t b}\right] d x d y,} \\
{\left[K_{t s}^{e}\right]=\int_{A}\left[B_{t s}\right]^{T}\left(\left[D_{t s}\right]+\left[D_{t s}\right]_{v}+\left[D_{t s}\right]_{p}\right)\left[B_{t s}\right] d x d y} \\
{\left[K_{t b s}^{e}\right]_{p b}=\int_{A}\left[B_{t b}\right]^{T}\left[D_{t b s}\right]_{p}\left[B_{t s}\right] d x d y,} \\
{\left[K_{t b s}^{e}\right]_{p s}=\int_{A}\left[B_{t s}\right]^{T}\left[D_{t b s}\right]_{p}\left[B_{t b}\right] d x d y,} \\
{\left[K_{t r b}^{e}\right]=\int_{A}\left[B_{t r b}\right]^{T}\left(\left[D_{t r b}\right]+\left[D_{t r b}\right]_{v}+\left[D_{t r b}\right]_{p}\right)} \\
\times\left[B_{t r b}\right] d x d y,
\end{gathered}
$$




$$
\begin{aligned}
& {\left[K_{t r b s}^{e}\right]_{p b}=\int_{A}\left[B_{t b}\right]^{T}\left[D_{t r b s}\right]_{p}\left[B_{r s}\right] d x d y,} \\
& {\left[K_{r t b s}^{e}\right]_{p b}=\int_{A}\left[B_{r b}\right]^{T}\left[D_{r t b s}\right]_{p}\left[B_{t s}\right] d x d y,} \\
& {\left[K_{t r b s}^{e}\right]_{p s}=\int_{A}\left[B_{t s}\right]^{T}\left[D_{r t b s}\right]_{p}\left[B_{r b}\right] d x d y,} \\
& {\left[K_{r t b s}^{e}\right]_{p s}=\int_{A}\left[B_{r s}\right]^{T}\left[D_{t r b s}\right]_{p}\left[B_{t b}\right] d x d y,} \\
& {\left[K_{t r s}^{e}\right]=\int_{A}\left[B_{t r s}\right]^{T}\left(\left[D_{t r s}\right]+\left[D_{t r s}\right]_{v}+\left[D_{t r s}\right]_{p}\right)} \\
& \times\left[B_{t r s}\right] d x d y, \\
& {\left[K_{r r b}^{e}\right]=\int_{A}\left[B_{r r b}\right]^{T}\left(\left[D_{r r b}\right]+\left[D_{r r b}\right]_{v}+\left[D_{r r b}\right]_{p}\right)} \\
& \times\left[B_{r r b}\right] d x d y, \\
& {\left[K_{r r s}^{e}\right]=\int_{A}\left[B_{r r s}\right]^{T}\left(\left[D_{r r s}\right]+\left[D_{r r s}\right]_{v}+\left[D_{r r s}\right]_{p}\right)} \\
& \times\left[B_{r r s}\right] d x d y, \\
& {\left[K_{r r b s}^{e}\right]_{p b}=\int_{A}\left[B_{r b}\right]^{T}\left[D_{r r b s}\right]_{p}\left[B_{r s}\right] d x d y,} \\
& {\left[K_{r r b s}^{e}\right]_{p s}=\int_{A}\left[B_{r s}\right]^{T}\left[D_{r r b s}\right]_{p}\left[B_{r b}\right] d x d y,} \\
& \left\{F_{t b}^{e}\right\}_{p}=\int_{A}\left[B_{t b}\right]^{T}\left\{D_{t b}\right\}_{p} d x d y, \\
& \left\{F_{t s}^{e}\right\}_{p}=\int_{A}\left[B_{t s}\right]^{T}\left\{D_{t s}\right\}_{p} d x d y, \\
& \left\{F_{r s}^{e}\right\}_{p}=\int_{A}\left[B_{r s}\right]^{T}\left\{D_{r s}\right\}_{p} d x d y .
\end{aligned}
$$

The various rigidity matrices and the rigidity vectors for electro-elastic coupling appearing in the previous elemental matrices are given by

$$
\begin{gathered}
{\left[D_{t b}\right]=\sum_{k=1}^{N} \int_{h_{k}}^{h_{k+1}}\left[\bar{C}_{b}^{k}\right] d z,} \\
{\left[D_{t r b}\right]=\sum_{k=1}^{N} \int_{h_{k}}^{h_{k+1}}\left[\bar{C}_{b}^{k}\right]\left[Z_{1}\right] d z,} \\
{\left[D_{r r b}\right]=\sum_{k=1}^{N} \int_{h_{k}}^{h_{k+1}}\left[Z_{1}\right]^{T}\left[\bar{C}_{b}^{k}\right]\left[Z_{1}\right] d z,} \\
{\left[D_{t s}\right]=\sum_{k=1}^{N} \int_{h_{k}}^{h_{k+1}}\left[\bar{C}_{s}^{k}\right] d z,} \\
{\left[D_{t r s}\right]=\sum_{k=1}^{N} \int_{h_{k}}^{h_{k+1}}\left[\bar{C}_{b}^{k}\right]\left[Z_{4}\right] d z,}
\end{gathered}
$$

$$
\begin{gathered}
{\left[D_{r r s}\right]=\sum_{k=1}^{N} \int_{h_{k}}^{h_{k+1}}\left[Z_{4}\right]^{T}\left[\bar{C}_{b}^{k}\right]\left[Z_{4}\right] d z,} \\
{\left[D_{t b}\right]_{v}=h_{v}\left[\bar{C}_{b}^{N+1}\right],} \\
{\left[D_{t r b}\right]_{v}=\int_{h_{N+1}}^{h_{N+2}}\left[\bar{C}_{b}^{N+1}\right]\left[Z_{2}\right] d z,}
\end{gathered}
$$$$
\left[D_{r r b}\right]_{v}=\int_{h_{N+1}}^{h_{N+2}}\left[Z_{2}\right]^{T}\left[\bar{C}_{b}^{N+1}\right]\left[Z_{2}\right] d z,
$$$$
\left[D_{t s}\right]_{v}=h_{v}\left[\bar{C}_{s}^{N+1}\right] \text {, }
$$$$
\left[D_{t r s}\right]_{v}=\int_{h_{N+1}}^{h_{N+2}}\left[\bar{C}_{s}^{N+1}\right]\left[Z_{5}\right] d z,
$$$$
\left[D_{r r s}\right]_{v}=\int_{h_{N+1}}^{h_{N+2}}\left[Z_{5}\right]^{T}\left[\bar{C}_{b}^{N+1}\right]\left[Z_{5}\right] d z,
$$$$
\left[D_{t b}\right]_{p}=h_{p}\left[\bar{C}_{b}^{N+2}\right] \text {, }
$$$$
\left[D_{t r b}\right]_{p}=\int_{h_{N+2}}^{h_{N+3}}\left[\bar{C}_{b}^{N+2}\right]\left[Z_{3}\right] d z,
$$$$
\left[D_{r r b}\right]_{p}=\int_{h_{N+2}}^{h_{N+3}}\left[Z_{3}\right]^{T}\left[\bar{C}_{b}^{N+2}\right]\left[Z_{3}\right] d z,
$$$$
\left[D_{t s}\right]_{p}=h_{p}\left[\bar{C}_{s}^{N+2}\right],
$$$$
\left[D_{t r s}\right]_{p}=\int_{h_{N+2}}^{h_{N+3}}\left[\bar{C}_{s}^{N+2}\right]\left[Z_{6}\right] d z,
$$$$
\left[D_{r r s}\right]_{p}=\int_{h_{N+2}}^{h_{N+3}}\left[Z_{6}\right]^{T}\left[\bar{C}_{b}^{N+2}\right]\left[Z_{6}\right] d z,
$$$$
\left[D_{t b s}\right]_{p}=\int_{h_{N+2}}^{h_{N+3}}\left[\bar{C}_{b s}^{N+2}\right] d z,
$$$$
\left[D_{t r b s}\right]_{p}=\int_{h_{N+2}}^{h_{N+3}}\left[\bar{C}_{b s}^{N+2}\right]\left[Z_{6}\right] d z,
$$$$
\left[D_{r t b s}\right]_{p}=\int_{h_{N+2}}^{h_{N+3}}\left[Z_{3}\right]^{T}\left[\bar{C}_{b s}^{N+2}\right] d z,
$$$$
\left[D_{r r b s}\right]_{p}=\int_{h_{N+2}}^{h_{N+3}}\left[Z_{3}\right]^{T}\left[\bar{C}_{b s}^{N+3}\right]\left[Z_{6}\right] d z,
$$$$
\left\{D_{t b}\right\}_{p}=\int_{h_{N+2}}^{h_{N+3}}-\frac{\left\{\bar{e}_{b}\right\}}{h_{p}} d z
$$$$
\left\{D_{r b}\right\}_{p}=\int_{h_{N+2}}^{h_{N+3}}-\frac{\left[Z_{3}\right]^{T}\left\{\bar{e}_{b}\right\}}{h_{p}} d z,
$$$$
\left\{D_{t s}\right\}_{p}=\int_{h_{N+2}}^{h_{N+3}}-\frac{\left\{\bar{e}_{s}\right\}}{h_{p}} d z,
$$$$
\left\{D_{r s}\right\}_{p}=\int_{h_{N+2}}^{h_{N+3}}-\frac{\left[Z_{6}\right]^{T}\left\{\bar{e}_{s}\right\}}{h_{p}} d z .
$$ 
Applying the dynamic version of the virtual work principle [32], the following open-loop equations of motion for an element of the overall plate integrated with the ACLD treatment are obtained:

$$
\begin{gathered}
{\left[M^{e}\right]\left\{\ddot{d}_{t}^{e}\right\}+\left[K_{t t}^{e}\right]\left\{d_{t}^{e}\right\}+\left[K_{t r}^{e}\right]\left\{d_{r}^{e}\right\}=\left\{F_{t p}^{e}\right\} V+\left\{F^{e}\right\},} \\
{\left[K_{t r}^{e}\right]^{T}\left\{d_{t}^{e}\right\}+\left[K_{r r}^{e}\right]\left\{d_{r}^{e}\right\}=\left\{F_{r p}^{e}\right\} V .}
\end{gathered}
$$

Since the elastic constant matrix of the viscoelastic layer is complex, the stiffness matrices of an element integrated with the ACLD treatment are complex. In case of an element not integrated with the ACLD treatment, the electro-elastic coupling matrices become null vectors and the elemental stiffness matrices are to be computed without considering the piezoelectric and viscoelastic layers. It should also be noted that as the stiffness matrices associated with the transverse shear strains are derived separately, one can employ the selective integration rule in a straight forward manner to avoid shear locking problem in case of thin plates. The restrained boundary conditions at the skew edges of the plate are such that the displacements of the points located at the skew edges are to be restrained along $x^{\prime}-, y^{\prime}$-, and $z^{\prime}$-directions (Figure 1(a)). Hence, in order to impose the boundary conditions in a straight forward manner, the generalized displacement vectors of a point lying on the skew edge are to be transformed as follows:

$$
\left\{d_{t}\right\}=\left[L_{t}\right]\left\{d_{t}^{\prime}\right\}, \quad\left\{d_{r}\right\}=\left[L_{r}\right]\left\{d_{r}^{\prime}\right\},
$$

where $\left\{d_{t}^{\prime}\right\}$ and $\left\{d_{r}^{\prime}\right\}$ are the generalized displacement vectors of the point with respect to $x^{\prime} y^{\prime} z^{\prime}$ coordinate system and are given by

$$
\begin{aligned}
& \left\{d_{t}^{\prime}\right\}=\left[\begin{array}{lll}
u_{o}^{\prime} & v_{o}^{\prime} & w_{o}^{\prime}
\end{array}\right]^{T}, \\
& \left\{d_{r}^{\prime}\right\}=\left[\begin{array}{lllllllll}
\theta_{x}^{\prime} & \theta_{y}^{\prime} & \theta_{z}^{\prime} & \phi_{x}^{\prime} & \phi_{y}^{\prime} & \phi_{z}^{\prime} & \gamma_{z}^{\prime} & \gamma_{y}^{\prime} & \gamma_{z}^{\prime}
\end{array}\right]^{T} \text {. }
\end{aligned}
$$

Also, the transformation matrices $\left[L_{t}\right]$ and $\left[L_{r}\right]$ are given by

$$
\begin{gathered}
{\left[L_{t}\right]=\left[\begin{array}{ccc}
c & s & 0 \\
-s & c & 0 \\
0 & 0 & 1
\end{array}\right],} \\
{\left[L_{r}\right]=\left[\begin{array}{ccccccccc}
c & s & 0 & 0 & 0 & 0 & 0 & 0 & 0 \\
-s & c & 0 & 0 & 0 & 0 & 0 & 0 & 0 \\
0 & 0 & 1 & 0 & 0 & 0 & 0 & 0 & 0 \\
0 & 0 & 0 & c & s & 0 & 0 & 0 & 0 \\
0 & 0 & 0 & -s & c & 0 & 0 & 0 & 0 \\
0 & 0 & 0 & 0 & 0 & 1 & 0 & 0 & 0 \\
0 & 0 & 0 & 0 & 0 & 0 & c & s & 0 \\
0 & 0 & 0 & 0 & 0 & 0 & -s & c & 0 \\
0 & 0 & 0 & 0 & 0 & 0 & 0 & 0 & 1
\end{array}\right],}
\end{gathered}
$$

in which $c=\cos \alpha$ and $s=\sin \alpha$. Thus, the elemental matrices of the element containing the nodes lying on the skew edge are to be augmented as follows:

$$
\begin{array}{ll}
{\left[\bar{K}_{t t}^{e}\right]=\left[T_{1}\right]^{T}\left[K_{t t}^{e}\right]\left[T_{1}\right],} & {\left[\bar{K}_{t r}^{e}\right]=\left[T_{1}\right]^{T}\left[K_{t r}^{e}\right]\left[T_{2}\right],} \\
{\left[\bar{K}_{r r}^{e}\right]=\left[T_{2}\right]^{T}\left[K_{r r}^{e}\right]\left[T_{2}\right],} & {\left[\bar{M}^{e}\right]=\left[T_{1}\right]^{T}\left[M^{e}\right]\left[T_{1}\right] .}
\end{array}
$$

The forms of the transformation matrices $\left[T_{1}\right]$ and $\left[T_{2}\right]$ are given by

$$
\begin{aligned}
{\left[T_{1}\right]=} & {\left[\begin{array}{cccccccc}
{\left[L_{t}\right]} & \widehat{o} & \widehat{o} & \widehat{o} & \widehat{o} & \widehat{o} & \widehat{o} & \widehat{o} \\
\widehat{o} & {\left[L_{t}\right]} & \widehat{o} & \widehat{o} & \widehat{o} & \widehat{o} & \widehat{o} & \widehat{o} \\
\widehat{o} & \widehat{o} & {\left[L_{t}\right]} & \widehat{o} & \widehat{o} & \widehat{o} & \widehat{o} & \widehat{o} \\
\widehat{o} & \widehat{o} & \widehat{o} & {\left[L_{t}\right]} & \widehat{o} & \widehat{o} & \widehat{o} & \widehat{o} \\
\widehat{o} & \widehat{o} & \widehat{o} & \widehat{o} & {\left[L_{t}\right]} & \widehat{o} & \widehat{o} & \widehat{o} \\
\widehat{o} & \widehat{o} & \widehat{o} & \widehat{o} & \widehat{o} & {\left[L_{t}\right]} & \widehat{o} & \widehat{o} \\
\widehat{o} & \widehat{o} & \widehat{o} & \widehat{o} & \widehat{o} & \widehat{o} & {\left[L_{t}\right]} & \widehat{o} \\
\widehat{o} & \widehat{o} & \widehat{o} & \widehat{o} & \widehat{o} & \widehat{o} & \widehat{o} & {\left[L_{t}\right]}
\end{array}\right], } \\
{\left[T_{2}\right]=} & {\left[\begin{array}{cccccccc}
{\left[L_{r}\right]} & \bar{o} & \bar{o} & \bar{o} & \bar{o} & \bar{o} & \bar{o} & \bar{o} \\
\bar{o} & {\left[L_{r}\right]} & \bar{o} & \bar{o} & \bar{o} & \bar{o} & \bar{o} & \bar{o} \\
\bar{o} & \bar{o} & {\left[L_{r}\right]} & \bar{o} & \bar{o} & \bar{o} & \bar{o} & \bar{o} \\
\bar{o} & \bar{o} & \bar{o} & {\left[L_{r}\right]} & \bar{o} & \bar{o} & \bar{o} & \bar{o} \\
\bar{o} & \bar{o} & \bar{o} & \bar{o} & {\left[L_{r}\right]} & \bar{o} & \bar{o} & \bar{o} \\
\bar{o} & \bar{o} & \bar{o} & \bar{o} & \bar{o} & {\left[L_{r}\right]} & \bar{o} & \bar{o} \\
\bar{o} & \bar{o} & \bar{o} & \bar{o} & \bar{o} & \bar{o} & {\left[L_{r}\right]} & \bar{o} \\
\bar{o} & \bar{o} & \bar{o} & \bar{o} & \bar{o} & \bar{o} & \bar{o} & {\left[L_{r}\right]}
\end{array}\right], }
\end{aligned}
$$

in which $\widehat{o}$ and $\bar{o}$ are $(3 \times 3)$ and $(9 \times 9)$ null matrices, respectively. It may be noted that in case of a node of such element which does not lie on the skew edge, the generalized displacement vectors of this node with respect to $x y z$ coordinate system are not required to be transformed. Hence, the matrices $\left[L_{t}\right]$ and $\left[L_{r}\right]$ corresponding to such node turn out to be $(3 \times 3)$ and $(9 \times 9)$ identity matrices, respectively.

The elemental equations of motion are now assembled to obtain the open-loop global equations of motion of the overall skew plate as follows:

$$
\begin{gathered}
{[M]\{\ddot{X}\}+\left[K_{t t}\right]\{X\}+\left[K_{t r}\right]\left\{X_{r}\right\}=\sum_{j=1}^{q}\left\{F_{t p}^{j}\right\} V^{j}+\{F\},} \\
{\left[K_{t r}\right]^{T}\{X\}+\left[K_{r r}\right]\left\{X_{r}\right\}=\sum_{j=1}^{q}\left\{F_{r p}^{j}\right\} V^{j},}
\end{gathered}
$$

where $\left[K_{t t}\right],\left[K_{t r}\right]$, and $\left[K_{r r}\right]$ are the global stiffness matrices of the overall skew laminated composite plate, $[M]$ is the global mass matrix, $\left\{F_{t p}\right\}$ and $\left\{F_{r p}\right\}$ are the global electroelastic coupling vectors, $\{X\}$ and $\left\{X_{r}\right\}$ are the global nodal generalized displacement vectors, $\{F\}$ is the global nodal force vector, $q$ is the number of patches, and $V^{j}$ is the voltage applied to the $j$ th patch. Since the elemental stiffness matrices of an element augmented with the ACLD treatment are complex, the global stiffness matrices become complex and the energy dissipation characteristics of the overall plate are attributed to the imaginary part of these matrices. Hence, the global equations of motion as derived previously also represent the modeling of the passive (uncontrolled) constrained layer damping of the substrate plate when the constraining layer is not subjected to any control voltage following a derivative control law. 


\section{Closed-Loop Model}

In the active control approach, the active constraining layer of each patch is activated with a control voltage negatively proportional to the transverse velocity of a point. Thus, the control voltage for each patch can be expressed in terms of the derivatives of the global nodal degrees of freedom as follows:

$$
V^{j}=-k_{d}^{j} \dot{w}=-k_{d}^{j}\left[U_{t}^{j}\right]\{\dot{X}\}-k_{d}^{j}\left(\frac{h}{2}\right)\left[U_{r}^{j}\right]\left\{\dot{X}_{r}\right\},
$$

where $\left[U_{t}^{j}\right]$ and $\left[U_{r}^{j}\right]$ are the unit vectors describing the location of the velocity sensor for the $j$ th patch and $k_{d}^{j}$ is the control gain for the $j$ th patch. Substituting (34) into (33), the final equations of motion governing the closedloop dynamics of the overall skew laminated composite plate/ACLD system can be obtained as follows:

$$
\begin{aligned}
& {[M]\{\ddot{X}\}+\left[K_{t t}\right]\{X\}+\left[K_{t r}\right]\left\{X_{r}\right\}+\sum_{j=1}^{m} k_{d}^{j}\left\{F_{t p}^{j}\right\}\left[U_{t}^{j}\right]\{\dot{X}\}} \\
& +\sum_{j=1}^{m} k_{d}^{j}\left(\frac{h}{2}\right)\left\{F_{t p}^{j}\right\}\left[U_{r}^{j}\right]\left\{\dot{X}_{r}\right\}=\{F\}, \\
& {\left[K_{t r}\right]^{T}\{X\}+\left[K_{r r}\right]\left\{X_{r}\right\}+\sum_{j=1}^{m} k_{d}^{j}\left\{F_{t p}^{j}\right\}\left[U_{t}^{j}\right]\{\dot{X}\}} \\
& +\sum_{j=1}^{m} k_{d}^{j}\left(\frac{h}{2}\right)\left\{F_{t p}^{j}\right\}\left[U_{r}^{j}\right]\left\{\dot{X}_{r}\right\}=0 .
\end{aligned}
$$

\section{Results and Discussion}

In this section, the numerical results obtained by the finite element model derived in the previous section have been presented. Symmetric as well as antisymmetric cross-ply and antisymmetric angle-ply laminated skew substrate plates integrated with the two patches of ACLD treatment are considered for presenting the numerical results. As shown in Figure 1, the locations of the patches have been selected in such a way that the energy dissipation corresponding to the first few modes becomes maximum. PZT-5H/spur composite with $60 \%$ fiber volume fraction has been considered for the material of the constraining layer of the ACLD treatment. Using the micromechanics model and the material properties of the constituent phases [35], the effective elastic and piezoelectric material properties of the 1-3 PZC with respect to the principle material coordinate system are computed as follows:

$$
\begin{gathered}
C_{11}=9.29 \mathrm{GPa}, \quad C_{12}=6.18 \mathrm{GPa}, \quad C_{13}=6.05 \mathrm{GPa}, \\
C_{33}=35.44 \mathrm{GPa}, \quad C_{23}=C_{13}, \quad C_{44}=1.58 \mathrm{GPa}, \\
C_{66}=1.54 \mathrm{GPa}, \quad C_{55}=C_{44},
\end{gathered}
$$

$$
\begin{gathered}
e_{31}=-0.1902 \mathrm{C} / \mathrm{m}^{2}, \quad e_{32}=e_{31}, \\
e_{33}=18.4107 \mathrm{C} / \mathrm{m}^{2}, \quad e_{24}=0.004 \mathrm{C} / \mathrm{m}^{2}, \\
e_{15}=e_{24} .
\end{gathered}
$$

The material of the orthotropic layers of the substrate plates is a graphite/epoxy composite and its material properties are [42]

$$
\begin{gathered}
E_{L}=172 \mathrm{GPa}, \quad \frac{E_{L}}{E_{T}}=25, \quad G_{L T}=0.5 E_{T}, \\
G_{T T}=0.2 E_{T}, \quad \nu_{L T}=\nu_{T T}=0.25,
\end{gathered}
$$

in which the symbols have the usual meaning. Unless otherwise mentioned, the aspect ratio $(a / h)$ and the thickness of the skew substrate plates are considered as 100 and $0.003 \mathrm{~m}$, respectively, while the orthotropic layers of the skew substrate plates are of equal thickness. Also, the length and the skew sides of the substrate are equal (i.e., $a=$ $b$ ). The loss factor of the viscoelastic layer considered for numerical results remains invariant [20] within a broad band $(0-400 \mathrm{~Hz})$ of frequency range, and the values of the complex shear modulus, Poisson ratio, and the density of the viscoelastic layer are considered as $20(1+i) \mathrm{MNm}^{-2}$, 0.49 , and $1140 \mathrm{~kg} / \mathrm{m}^{-3}$, respectively [20]. The thicknesses of the viscoelastic layer and the 1-3 PZC layer are considered as $50.8 \mu \mathrm{m}$ and $250 \mu \mathrm{m}$, respectively. The value of the shear correction factor is used as 5/6. Three points of Gaussian integration rule are considered for computing the element matrices corresponding to the bending deformations while two points of that are used for computing the element matrices corresponding to transverse shear deformations. The simply supported boundary conditions at the edges of the overall plate are invoked as follows:

$$
\begin{aligned}
& \text { at } x=y \tan \alpha, \quad x=a+y \tan \alpha: \\
& \quad v_{o}^{\prime}=w_{o}^{\prime}=\theta_{y}^{\prime}=\phi_{y}^{\prime}=\gamma_{y}^{\prime}=\theta_{z}^{\prime}=\gamma_{z}^{\prime}=0 \\
& \text { at } y=0, \quad y=b \cos \alpha: \\
& \quad u_{o}=w_{o}=\theta_{x}=\phi_{x}=\gamma_{x}=\theta_{z}=\gamma_{z}=0 .
\end{aligned}
$$

To verify the validity of the present finite element model (FEM), the natural frequencies of the skew laminated composite plates integrated with the inactivated patches of negligible thickness are first computed and subsequently compared with the existing analytical results [41] of the identical plates without being integrated with the patches. Such comparisons for the simply supported and clampedclamped skew laminated plates are presented in Tables 1 and 2 , respectively. It may be observed from these tables that the results are in excellent agreement, validating the model of the plate derived here.

In order to assess the performance of the vertically/obliquely reinforced 1-3 PZC as the material of the distributed actuators of smart skew laminated composite plates, frequency responses for ACLD of the substrate skew laminated composite plates are investigated. To compute the 
TABLE 1: Comparison of fundamental natural frequencies $(\bar{\omega})$ of simply supported skew laminated composite plates integrated with the patches of negligible thickness.

\begin{tabular}{|c|c|c|c|c|c|c|c|c|c|c|}
\hline \multirow[t]{2}{*}{ Skew angle } & \multirow[t]{2}{*}{ Source } & \multicolumn{3}{|c|}{$\begin{array}{c}\text { Symmetric cross-ply } \\
\left(90^{\circ} / 0^{\circ} / 90^{\circ} / 0^{\circ} / 90^{\circ}\right) \\
\text { Modes }\end{array}$} & \multicolumn{3}{|c|}{$\begin{array}{c}\text { Antisymmetric cross-ply } \\
\left(0^{\circ} / 90^{\circ} / 0^{\circ} / 90^{\circ}\right) \\
\text { Modes }\end{array}$} & \multicolumn{3}{|c|}{$\begin{array}{c}\text { Antisymmetric angle-ply } \\
\left(45^{\circ} /-45^{\circ} / 45^{\circ} /-45^{\circ}\right) \\
\text { Modes }\end{array}$} \\
\hline & & 1 & 2 & 3 & 1 & 2 & 3 & 1 & 2 & 3 \\
\hline \multirow{2}{*}{$0^{\circ}$} & Reference [41] & 1.5699 & 2.8917 & 3.7325 & 1.4829 & 2.4656 & 3.2522 & 1.7974 & 3.3351 & 3.3351 \\
\hline & Present FEM & 1.5635 & 2.4383 & 3.5033 & 1.5076 & 2.4380 & 3.2254 & 1.8493 & 3.3359 & 3.3370 \\
\hline \multirow{2}{*}{$15^{\circ}$} & Reference [41] & 1.6874 & 3.0458 & 3.9600 & 1.5741 & 2.5351 & 3.0270 & 1.8313 & 3.2490 & 3.6724 \\
\hline & Present FEM & 1.6571 & 2.9840 & 3.6505 & 1.5796 & 2.5775 & 2.9892 & 1.8675 & 3.2075 & 3.5810 \\
\hline \multirow{2}{*}{$30^{\circ}$} & Reference [41] & 2.0840 & 3.4023 & 4.6997 & 1.8871 & 2.9372 & 3.4489 & 2.0270 & 3.4431 & 4.2361 \\
\hline & Present FEM & 1.9596 & 3.1690 & 4.6796 & 1.8226 & 2.9585 & 3.2357 & 1.9894 & 3.2365 & 4.3208 \\
\hline \multirow{2}{*}{$45^{\circ}$} & Reference [41] & 2.8925 & 4.1906 & 5.4149 & 2.5609 & 3.3126 & 4.0617 & 2.5609 & 3.3131 & 4.2772 \\
\hline & Present FEM & 2.4811 & 4.4875 & 5.3289 & 2.2996 & 3.4773 & 4.4889 & 2.3194 & 3.4870 & 4.5009 \\
\hline
\end{tabular}

${ }^{*} \bar{\omega}=\left(\omega a^{2} / \pi^{2} h\right) \sqrt{\rho / E_{T}} ; \omega$ is the circular natural frequency.

TABLE 2: Comparison of fundamental natural frequencies $(\bar{\omega})$ of clamped-clamped skew laminated composite plates integrated with the patches of negligible thickness.

\begin{tabular}{|c|c|c|c|c|c|c|c|c|c|c|}
\hline \multirow[t]{2}{*}{ Skew angle } & \multirow[t]{2}{*}{ Source } & \multicolumn{3}{|c|}{$\begin{array}{c}\text { Symmetric cross-ply } \\
\left(90^{\circ} / 0^{\circ} / 90^{\circ} / 0^{\circ} / 90^{\circ}\right) \\
\text { Modes }\end{array}$} & \multicolumn{3}{|c|}{$\begin{array}{c}\text { Antisymmetric cross-ply } \\
\left(0^{\circ} / 90^{\circ} / 0^{\circ} / 90^{\circ}\right) \\
\text { Modes }\end{array}$} & \multicolumn{3}{|c|}{$\begin{array}{c}\text { Antisymmetric angle-ply } \\
\left(45^{\circ} /-45^{\circ} / 45^{\circ} /-45^{\circ}\right) \\
\text { Modes }\end{array}$} \\
\hline & & 1 & 2 & 3 & 1 & 2 & 3 & 1 & 2 & 3 \\
\hline \multirow{2}{*}{$0^{\circ}$} & Reference [41] & 2.3687 & 3.5399 & 4.1122 & 2.2990 & 3.7880 & 3.7880 & 2.2119 & 3.7339 & 3.7339 \\
\hline & Present FEM & 2.3201 & 3.4769 & 4.4102 & 2.3315 & 3.6531 & 3.6545 & 2.2433 & 3.6000 & 3.6012 \\
\hline \multirow{2}{*}{$15^{\circ}$} & Reference [41] & 2.4663 & 3.6255 & 4.3418 & 2.3809 & 3.7516 & 4.0785 & 2.3099 & 3.6997 & 4.0438 \\
\hline & Present FEM & 2.3699 & 3.4821 & 4.4049 & 2.3741 & 3.5856 & 3.8401 & 2.3049 & 3.5346 & 3.8092 \\
\hline \multirow{2}{*}{$30^{\circ}$} & Reference [41] & 2.7921 & 3.9557 & 5.0220 & 2.6666 & 3.9851 & 4.7227 & 2.6325 & 3.9549 & 5.2107 \\
\hline & Present FEM & 2.5366 & 3.5696 & 4.4734 & 2.5240 & 4.1943 & 4.5373 & 2.4945 & 3.6113 & 5.0932 \\
\hline \multirow{2}{*}{$45^{\circ}$} & Reference [41] & 3.4739 & 4.7129 & 5.8789 & 3.3015 & 4.6290 & 5.8423 & 3.3015 & 4.6290 & 5.8423 \\
\hline & Present FEM & 2.8665 & 4.7074 & 5.4562 & 2.8377 & 4.7614 & 5.6620 & 2.8377 & 4.7614 & 5.5162 \\
\hline
\end{tabular}

frequency response functions, the skew laminated composite plates are harmonically excited by a force of amplitude 1 Newton applied to a point $(a / 2,(a / 4) \cos \alpha, h / 2)$ on the skew substrate plates. The control voltage supplied to the constraining layer of the first patch is negatively proportional to the velocity of the point $(a / 2,(a / 4) \cos \alpha, h / 2)$ and that applied to the constraining layer of the second patch is negatively proportional to the velocity of the point $(a / 2,(3 a / 4) \cos \alpha, h / 2)$.

Figures 3, 4, and 5 illustrate the frequency response functions for the transverse displacement of a point of a simply supported symmetric cross-ply $\left(0^{\circ} / 90^{\circ} / 0^{\circ}\right)$ plate with $15^{\circ}, 30^{\circ}$, and $45^{\circ}$ skew angles, respectively, while the fiber orientation angle $(\psi)$ in the constraining layer is $0^{\circ}$. These figures display both uncontrolled and controlled responses and clearly show that the active constraining layer made of the vertically reinforced 1-3 PZC material being studied here significantly attenuates the amplitude of vibrations, enhancing the damping characteristics of the overall laminated skew composite plates over the passive damping (uncontrolled). Similar responses are also obtained for antisymmetric cross-ply $\left(0^{\circ} / 90^{\circ} / 0^{\circ} / 90^{\circ}\right)$ and antisymmetric angleply $\left(45^{\circ} /-45^{\circ} / 45^{\circ} /-45^{\circ}\right)$ skew laminated composite plates.
However, for brevity these results are not presented here. The maximum values of the control voltages required to compute the controlled responses presented in Figures 3-5 have been found to be quite low and are illustrated in Figure 6 in case of the symmetric cross-ply $\left(0^{\circ} / 90^{\circ} / 0^{\circ}\right)$ plate with skew angle $15^{\circ}$ only. In order to examine the contribution of the vertical actuation in improving the damping characteristics of the skew laminated composite plates as demonstrated in Figures 3-5, active control responses of the symmetric cross-ply laminated composite plate with $15^{\circ}$ skew angle are plotted in Figure 7 with and without considering the value of $e_{33}$ and $e_{31}$. It may be noted that when the values of $e_{31}$ is zero and that of $e_{33}$ is nonzero, the vertical actuation of the active constraining layer of the ACLD treatment is responsible for increasing the transverse shear deformations of the viscoelastic constrained layer over the passive counterpart resulting in improved damping of the smart plate over its passive damping. On the other hand, if $e_{33}$ is zero and $e_{31}$ is nonzero, the inplane actuation of the active constraining layer causes the transverse shear deformation of the viscoelastic core of the ACLD treatment leading to the active damping of the smart plate. It is evident from Figure 7 that the contribution of the 


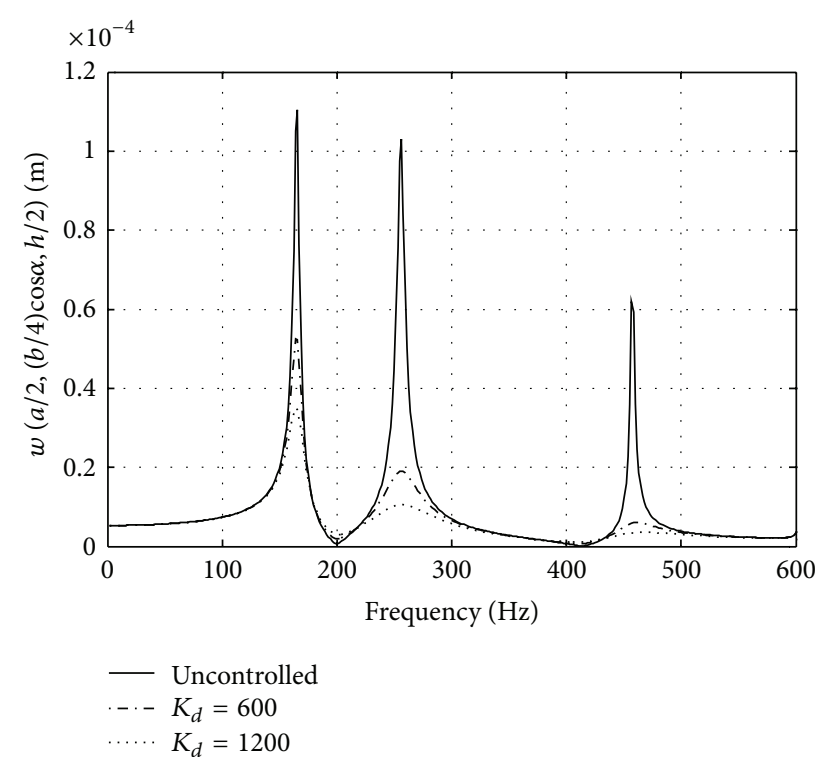

FIGURE 3: Frequency response functions for the transverse displacement $w(a / 2,(a / 4) \cos \alpha, h / 2)$ of a simply supported skew symmetric cross-ply $\left(0^{\circ} / 90^{\circ} / 0^{\circ}\right)$ plate $\left(a / h=100, \psi=0^{\circ}, \alpha=15^{\circ}\right)$.

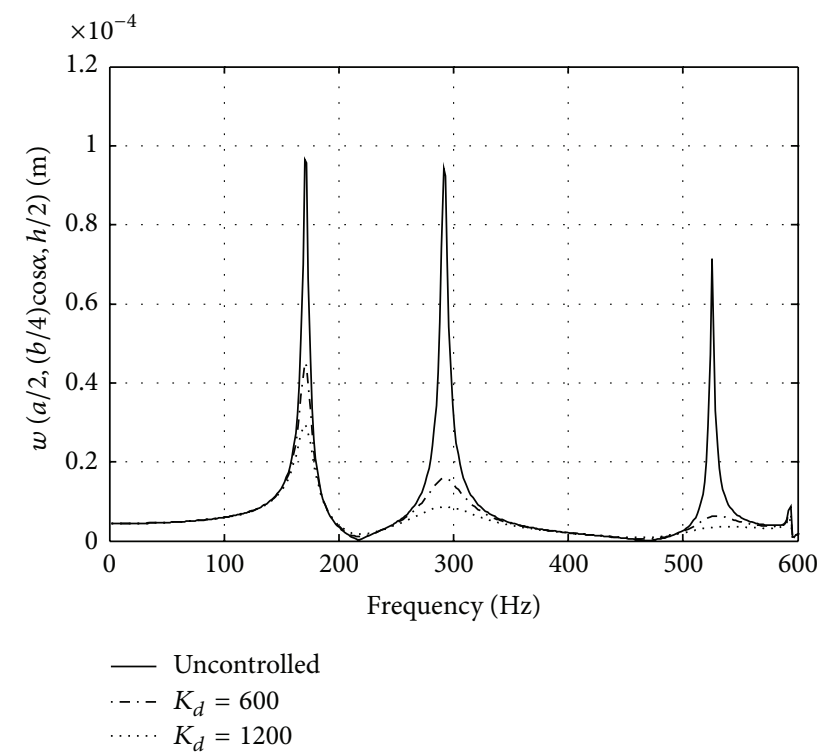

FIGURE 4: Frequency response functions for the transverse displacement $w(a / 2,(a / 4) \cos \alpha, h / 2)$ of a simply supported skew symmetric cross-ply $\left(0^{\circ} / 90^{\circ} / 0^{\circ}\right)$ plate $\left(a / h=100, \psi=0^{\circ}, \alpha=30^{\circ}\right)$.

vertical actuation by the constraining vertically reinforced $1-$ 3 PZC layer is significantly larger than that of the in-plane actuation by the same for controlling the modes displayed in Figure 7. Though not shown here, similar results are also obtained for antisymmetric cross-ply and angle-ply skew plates.

Figures 8, 9, and 10 demonstrate the effect of variation of piezoelectric fiber orientation angle on the performance of the patches for improving the damping characteristics of simply supported symmetric cross-ply $\left(0^{\circ} / 90^{\circ} / 0^{\circ}\right)$,

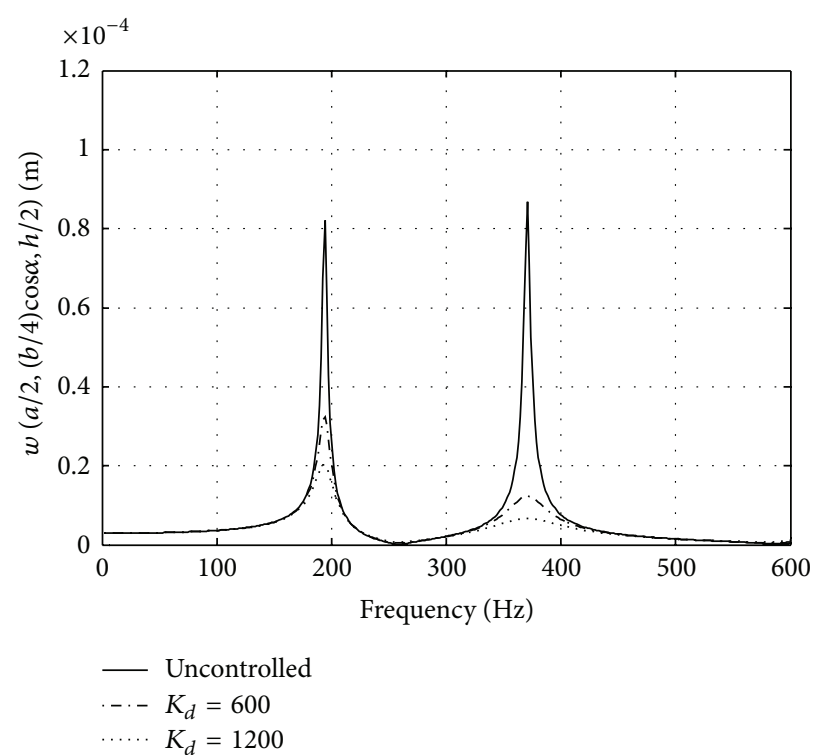

FIGURE 5: Frequency response functions for the transverse displacement $w(a / 2,(a / 4) \cos \alpha, h / 2)$ of a simply supported skew symmetric cross-ply $\left(0^{\circ} / 90^{\circ} / 0^{\circ}\right)$ plate $\left(a / h=100, \psi=0^{\circ}, \alpha=45^{\circ}\right)$.

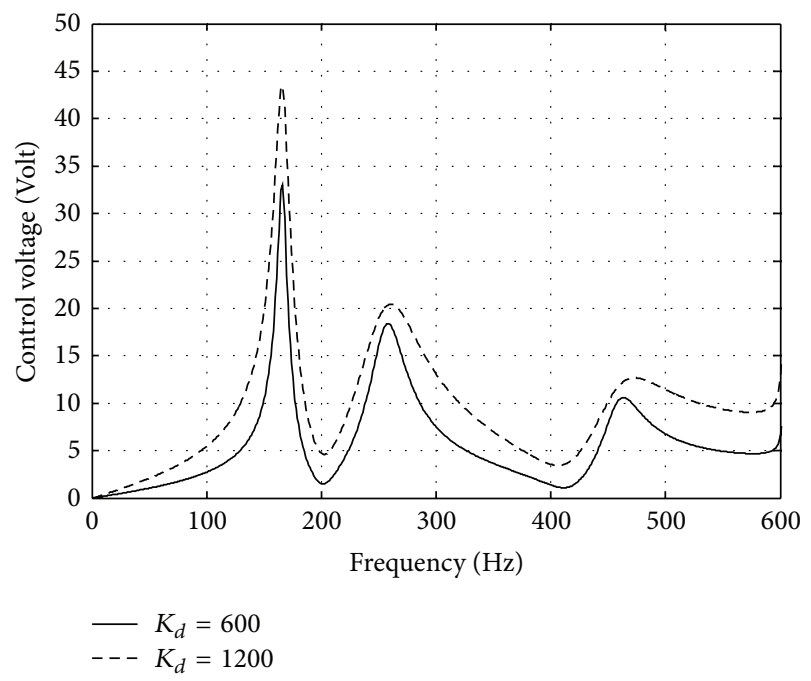

FIGURE 6: Frequency response functions for the control voltages for active damping of the simply supported skew symmetric cross-ply $\left(0^{\circ} / 90^{\circ} / 0^{\circ}\right)$ plate $\left(a / h=100, \psi=0^{\circ}, \alpha=15^{\circ}\right)$.

antisymmetric cross-ply $\left(0^{\circ} / 90^{\circ} / 0^{\circ} / 90^{\circ}\right)$, and angle-ply $\left(45^{\circ} /-45^{\circ} / 45^{\circ} /-45^{\circ}\right)$ substrate skew composite plates with $30^{\circ}$ skew angle, respectively, while the orientation angle $(\psi)$ of the piezoelectric fibers is varied in the vertical $x z$-plane. These figures illustrate that the attenuating capability of the patches becomes maximum when the value of the orientation angle $(\psi)$ of the fibers in the $x z$-plane is $0^{\circ}$. Similar effect is also obtained when the fiber orientation angle $(\psi)$ in the constraining layer of the ACLD treatment is varied in the vertical $y z$-plane as illustrated in Figures 11, 12, and 13 for the symmetric cross-ply $\left(0^{\circ} / 90^{\circ} / 0^{\circ}\right)$, antisymmetric cross-ply $\left(0^{\circ} / 90^{\circ} / 0^{\circ} / 90^{\circ}\right)$, and angle-ply $\left(45^{\circ} /-45^{\circ} / 45^{\circ} /-45^{\circ}\right)$ substrate 


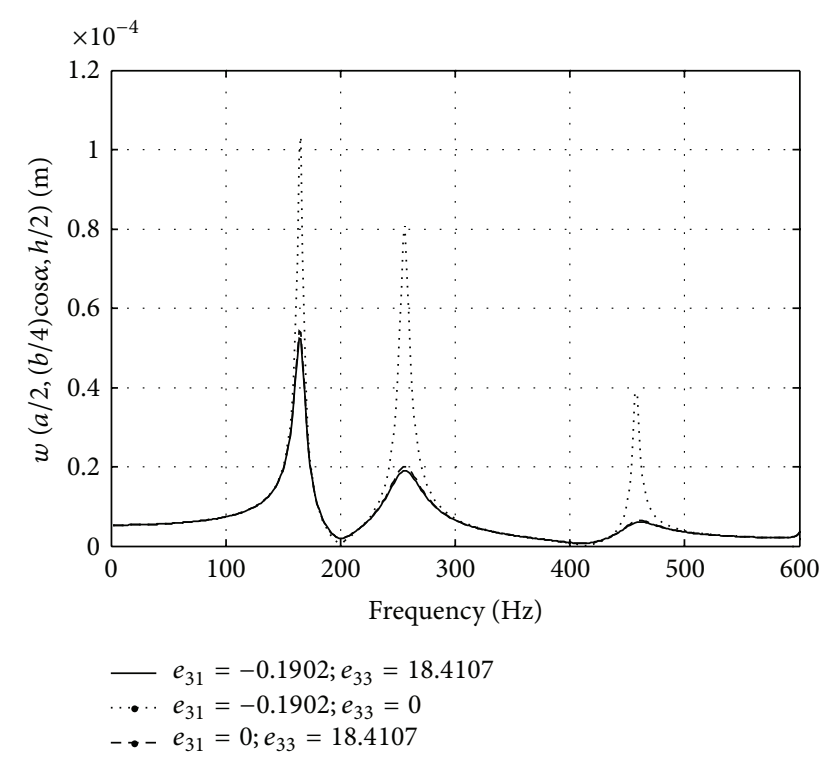

FIGURE 7: Frequency response functions demonstrating the contribution of vertical actuation on the controlled responses for the transverse displacement $w(a / 2,(a / 4) \cos \alpha, h / 2)$ of the skew symmetric cross-ply $\left(0^{\circ} / 90^{\circ} / 0^{\circ}\right)$ plate $\left(K_{d}=600, a / h=100, \psi=0^{\circ}\right.$, $\alpha=15^{\circ}$ ).

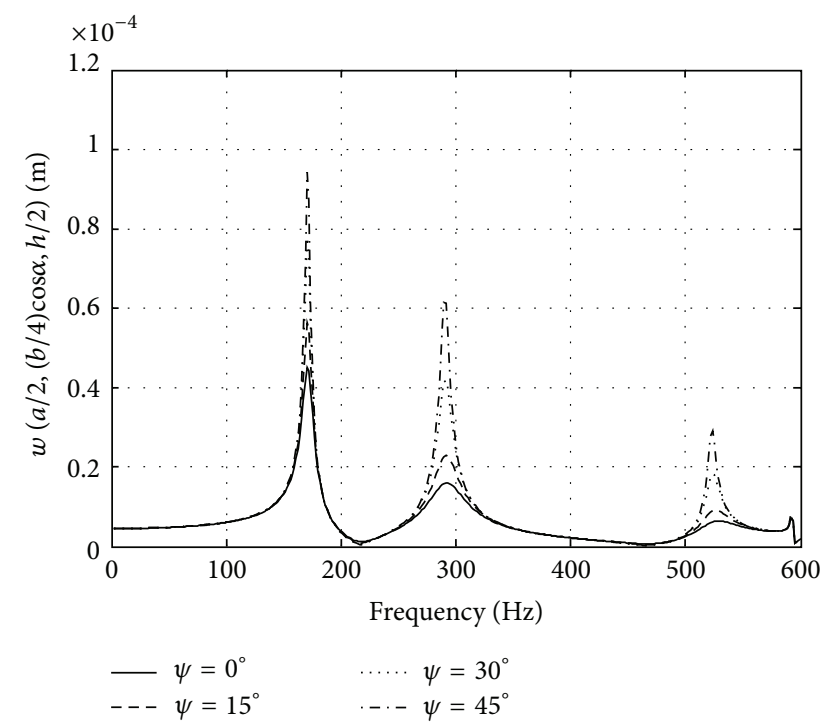

FIGURE 8: Effect of variation of piezoelectric fiber orientation angle $(\psi)$ on the controlled responses of a simply supported symmetric skew cross-ply $\left(0^{\circ} / 90^{\circ} / 0^{\circ}\right)$ plate when the piezoelectric fibers of the constraining layer are coplanar with the $x z$-plane $\left(K_{d}=600, a / h=\right.$ $100, \alpha=30^{\circ}$ ).

skew composite plates with $30^{\circ}$ skew angle, respectively. In this regard, it should be noted that for investigating the effect of variation of piezoelectric fiber orientation angle $(\psi)$ on the performance of the ACLD patches, the value of $\psi$ has been smoothly varied to compute the controlled responses. However, for the sake of clarity in the plots, the responses corresponding to the four specific values of $\psi$

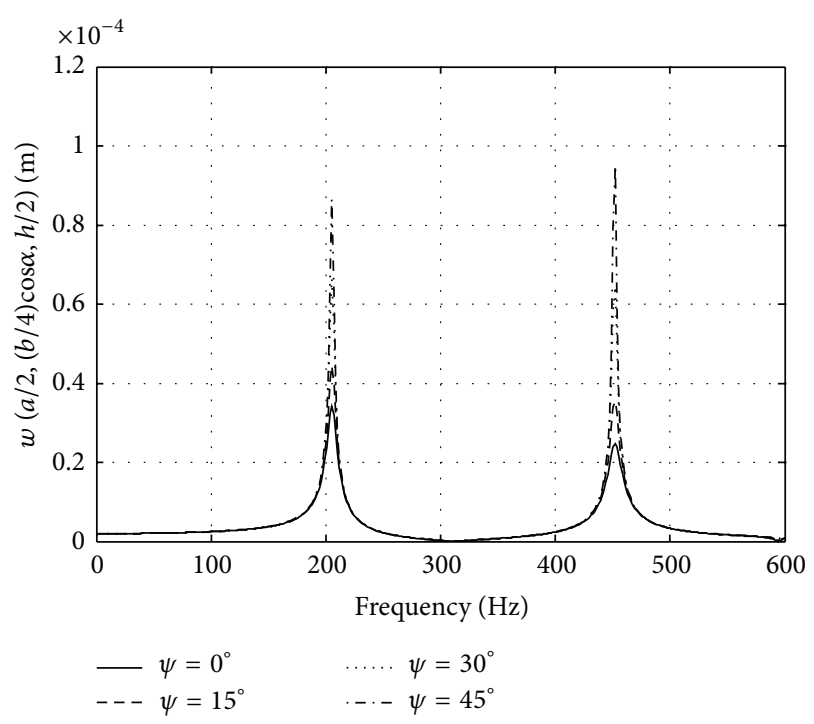

FIGURE 9: Effect of variation of piezoelectric fiber orientation angle $(\psi)$ on the controlled responses of a simply supported skew antisymmetric cross-ply $\left(0^{\circ} / 90^{\circ} / 0^{\circ} / 90^{\circ}\right)$ plate when the piezoelectric fibers are coplanar with the $x z$-plane $\left(K_{d}=600, a / h=100\right.$, $\alpha=30^{\circ}$ ).

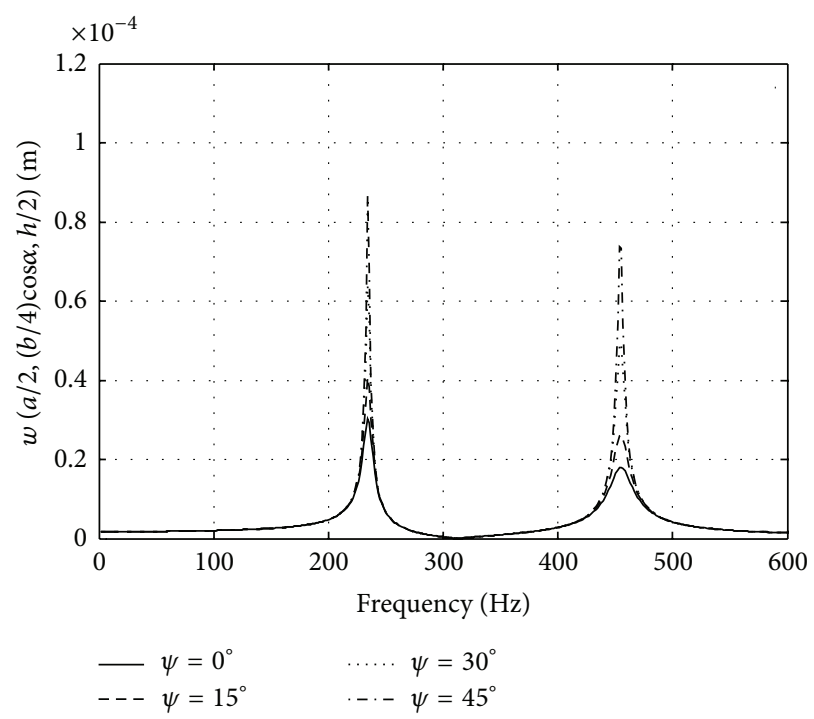

FIGURE 10: Effect of variation of piezoelectric fiber orientation angle $(\psi)$ on the controlled responses of a simply supported skew antisymmetric angle-ply $\left(45^{\circ} /-45^{\circ} / 45^{\circ} /-45^{\circ}\right)$ plate when the piezoelectric fibers are coplanar with the $x z$-plane $\left(K_{d}=600, a / h=\right.$ $100, \alpha=30^{\circ}$ ).

have been presented such that the optimum performance of the patches can be evaluated. The significant effect of the variation of the piezoelectric fiber orientation angle in the vertical $x z$ - and $y z$-planes has been noticed when all the boundaries of the overall plates are clamped as shown in Figures 14-19. When the piezoelectric fibers are coplanar with the vertical $x z$-plane, the frequency response functions plotted in Figures 14, 15, and 16 reveal that the control 


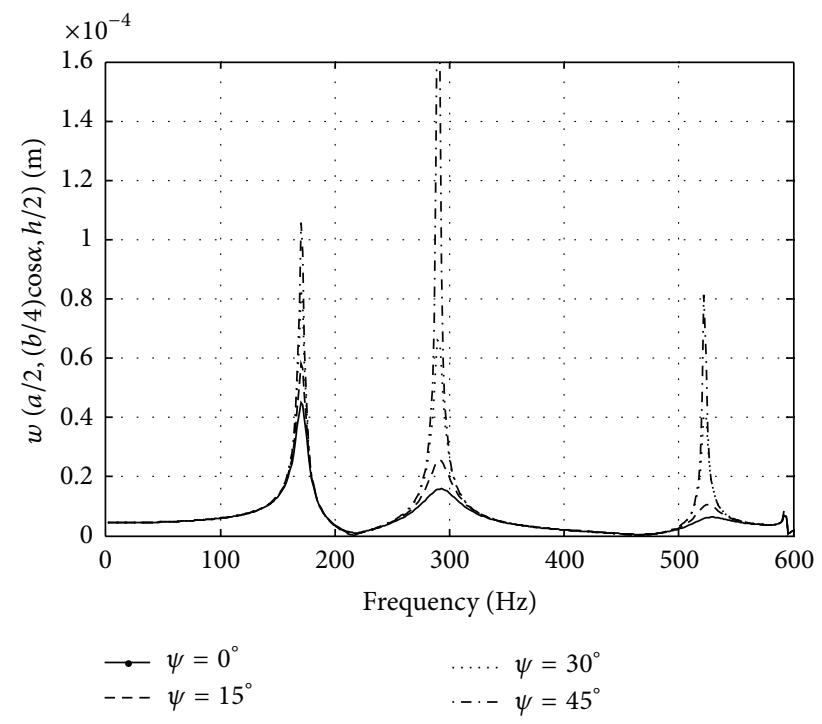

FIGURE 11: Effect of variation of piezoelectric fiber orientation angle $(\psi)$ on the controlled responses of a simply supported skew symmetric cross-ply $\left(0^{\circ} / 90^{\circ} / 0^{\circ}\right)$, plate when the piezoelectric fibers of the constraining layer are coplanar with the $y z$-plane $\left(K_{d}=600\right.$, $\left.a / h=100, \alpha=30^{\circ}\right)$.

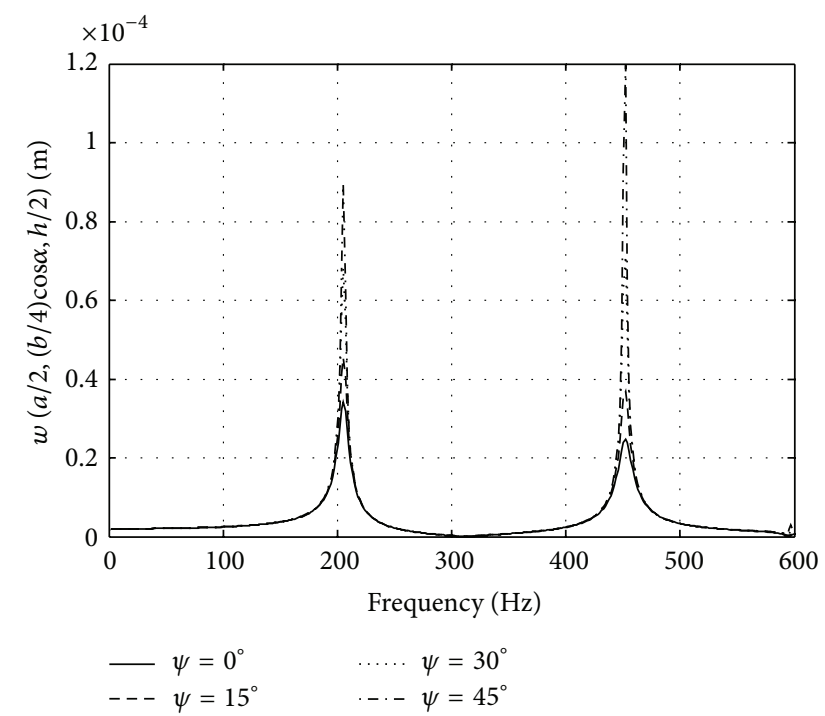

FIGURE 12: Effect of variation of piezoelectric fiber orientation angle $(\psi)$ on the controlled responses of a simply supported skew antisymmetric cross-ply $\left(0^{\circ} / 90^{\circ} / 0^{\circ} / 90^{\circ}\right)$ plate when the piezoelectric fibers of the constraining layer are coplanar with the $y z$-plane $\left(K_{d}=\right.$ $600, a / h=100, \alpha=30^{\circ}$ ).

authority of the patches becomes maximum for improving the damping characteristics of the clamped-clamped skew cross-ply and angle-ply substrate plates if the value of the piezoelectric fiber orientation angle $(\psi)$ is $30^{\circ}$. The same is also found to be true if the piezoelectric fibers are coplanar with the vertical $y z$-plane as shown in Figures 17, 18, and 19 for the previously mentioned skew plates. Similar responses are also found for the effect of piezoelectric fiber orientation

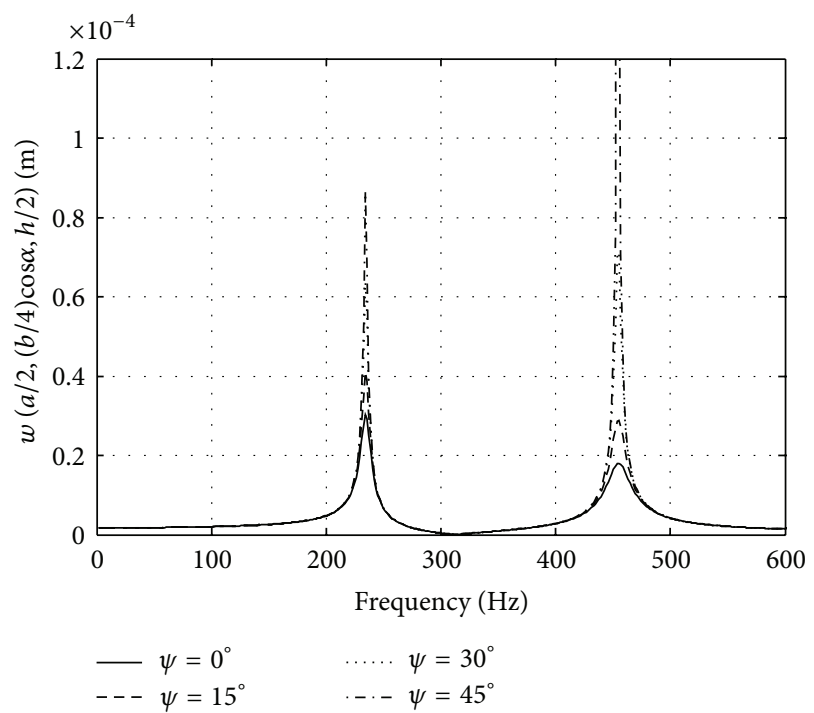

FIGURE 13: Effect of variation of piezoelectric fiber orientation angle $(\psi)$ on the controlled responses of a simply supported skew antisymmetric angle-ply $\left(45^{\circ} /-45^{\circ} / 45^{\circ} /-45^{\circ}\right)$ plate when the piezoelectric fibers of the constraining layer are coplanar with the $y z$-plane $\left(K_{d}=600, a / h=100, \alpha=30^{\circ}\right)$.

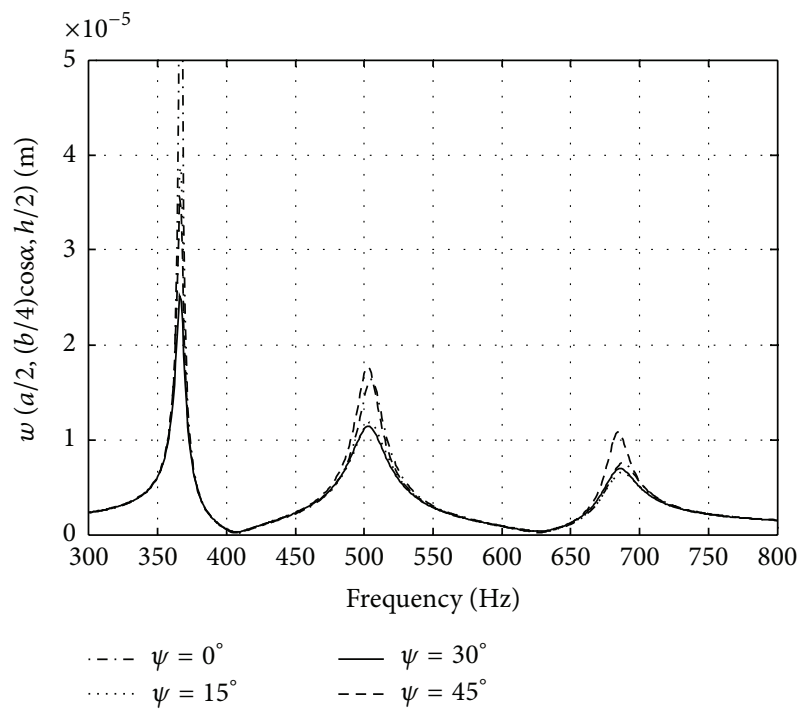

FIGURE 14: Effect of variation of piezoelectric fiber orientation angle $(\psi)$ on the controlled responses of a clamped-clamped skew symmetric cross-ply $\left(0^{\circ} / 90^{\circ} / 0^{\circ}\right)$ plate when the piezoelectric fibers of the constraining layer are coplanar with the $x z$-plane $\left(K_{d}=600\right.$, $a / h=100, \alpha=30^{\circ}$ ).

angle on the active damping of other skew laminated plates with different skew angles. However, for the sake of brevity, they are not presented here.

\section{Conclusions}

In this paper, a study has been carried out to investigate the performance of the ACLD treatment for active 


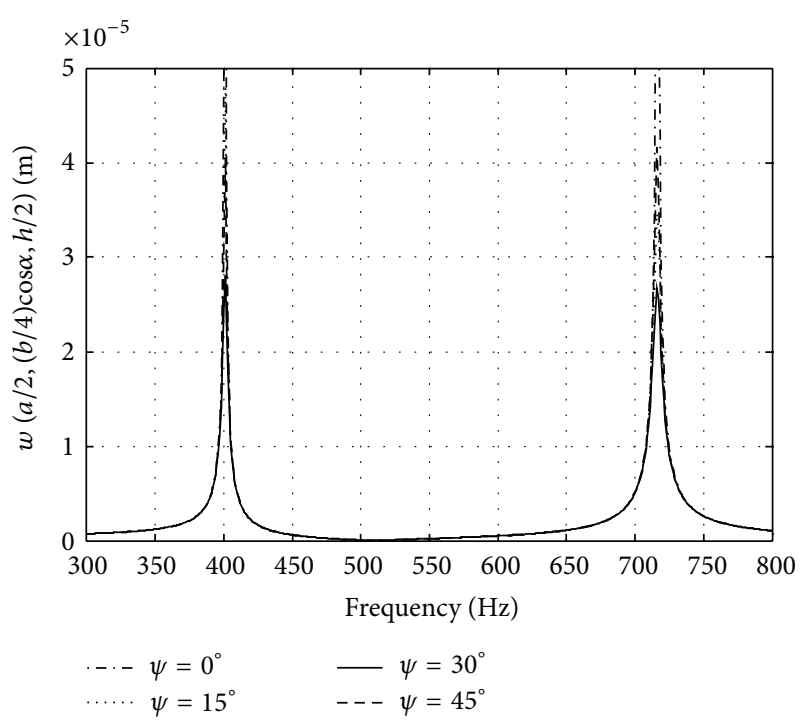

FIGURE 15: Effect of variation of piezoelectric fiber orientation angle $(\psi)$ on the controlled responses of a clamped-clamped skew antisymmetric cross-ply $\left(0^{\circ} / 90^{\circ} / 0^{\circ} / 90^{\circ}\right)$ plate when the piezoelectric fibers of the constraining layer are coplanar with the $x z$-plane $\left(K_{d}=\right.$ $600, a / h=100, \alpha=30^{\circ}$ ).

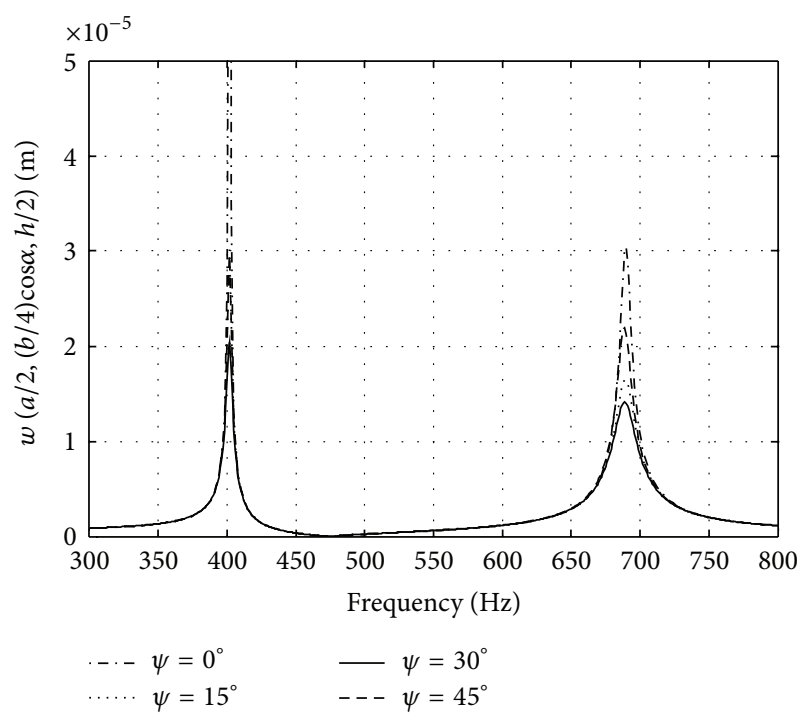

FIGURE 16: Effect of variation of piezoelectric fiber orientation angle $(\psi)$ on the controlled responses of a clamped-clamped skew antisymmetric angle-ply $\left(45^{\circ} /-45^{\circ} / 45^{\circ} /-45^{\circ}\right)$ plate when the piezoelectric fibers of the constraining layer are coplanar with the $x z$ plane $\left(K_{d}=600, a / h=100, \alpha=30^{\circ}\right)$.

damping of smart skew laminated composite plates when vertically/obliquely reinforced 1-3 PZC materials are used as the materials for the constraining layer of the ACLD treatment. A layerwise FSDT-based finite element model has been developed to describe the dynamics of the skew laminated composite plates integrated with the patches of ACLD treatment. Unlike the existing finite element models of smart structures integrated with ACLD treatment, the

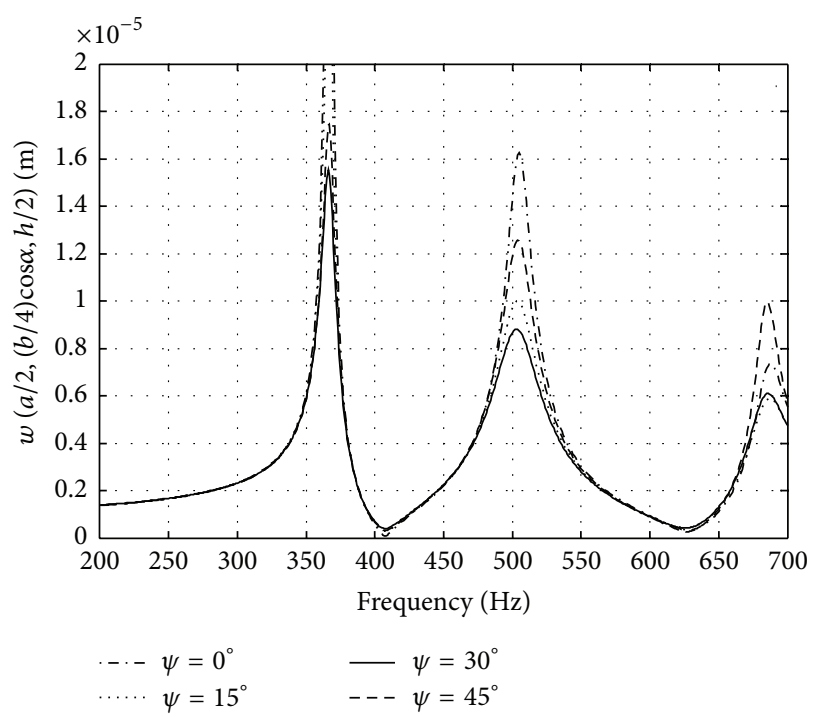

FIGURE 17: Effect of variation of piezoelectric fiber orientation angle $(\psi)$ on the controlled responses of a clamped-clamped skew symmetric cross-ply $\left(0^{\circ} / 90^{\circ} / 0^{\circ}\right)$ plate when the piezoelectric fibers of the constraining layer are coplanar with the $y z$-plane $\left(K_{d}=600\right.$, $\left.a / h=100, \alpha=30^{\circ}\right)$.

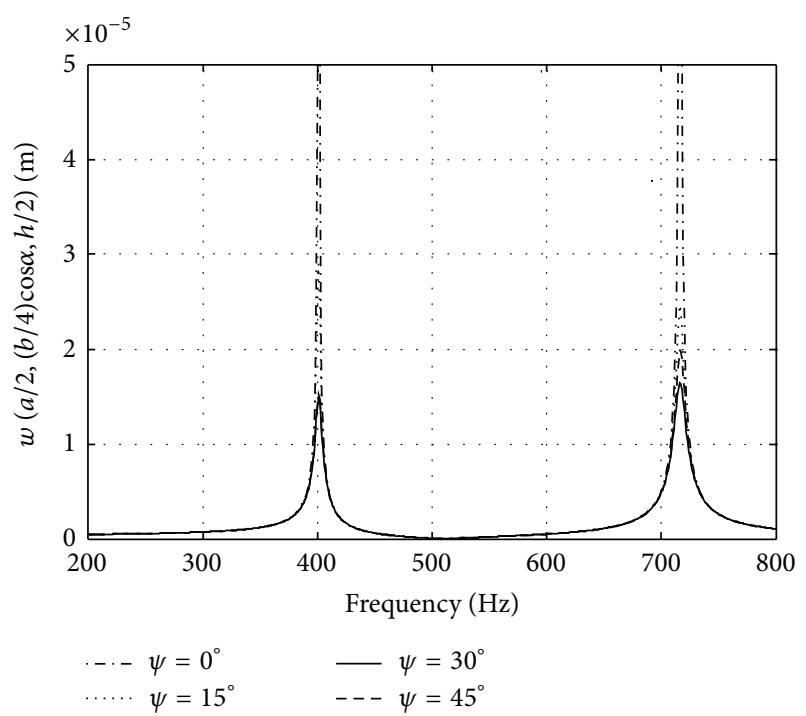

FIGURE 18: Effect of variation of piezoelectric fiber orientation angle $(\psi)$ on the controlled responses of a clamped-clamped skew antisymmetric cross-ply $\left(0^{\circ} / 90^{\circ} / 0^{\circ} / 90^{\circ}\right)$ plate when the piezoelectric fibers of the constraining layer are coplanar with the $y z$-plane $\left(K_{d}=\right.$ $600, a / h=100, \alpha=30^{\circ}$ ).

derivation of the present finite element model includes the transverse deformations of the substrate skew laminated composite plate, the constrained viscoelastic layer, and the constraining 1-3 PZC layer of the ACLD treatment along the thickness (i.e., $z$ ) direction such that both vertical and inplane actuations by the constraining layer of the patches can be utilized for active damping of the plates. The frequency 


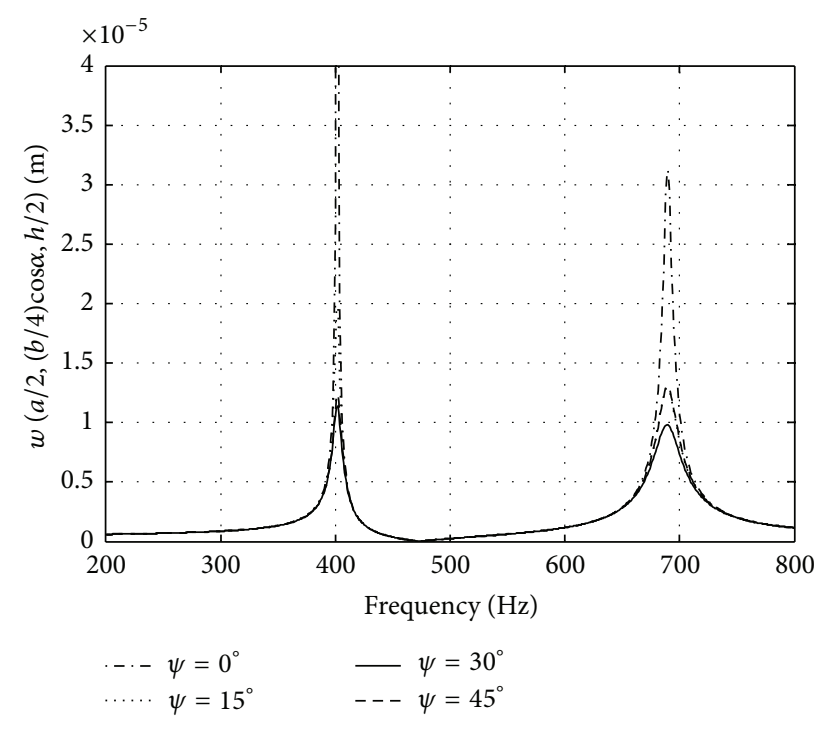

FIGURE 19: Effect of variation of piezoelectric fiber orientation angle $(\psi)$ on the controlled responses of a clamped-clamped skew antisymmetric angle-ply $\left(45^{\circ} /-45^{\circ} / 45^{\circ} /-45^{\circ}\right)$ plate when the piezoelectric fibers of the constraining layer are coplanar with the $y z$ plane $\left(K_{d}=600, a / h=100, \alpha=30^{\circ}\right)$.

responses of the symmetric cross-ply, antisymmetric crossply, and angle-ply skew composite plates indicate that the active constraining layer of the ACLD treatment being made of the vertically reinforced 1-3 PZC material significantly enhances the damping characteristics of the plates over the passive damping. The analysis revealed that if the vertically reinforced 1-3 PZC material is used for the constraining layer of the ACLD treatment, then the contribution of the vertical actuation by the constraining layer alone for improving the active damping characteristics of the smart skew laminated composite plate is significantly larger than that due to the in-plane actuation by the constraining layer alone. It is important to note from the present investigation that the variation of the orientation angle of the piezoelectric fibers of the constraining layer in the vertical $x z$ - and $y z$-planes as well as the skew angle and boundary conditions of the overall plates significantly affects the performance of the patches. For the simply supported laminated substrate plates, the performance of the patches becomes maximum when the piezoelectric fiber orientation angle $(\psi)$ is $0^{\circ}$, while in case of the clamped-clamped laminated skew substrate plates, the maximum control authority of the patches is achieved when the value of $\psi$ is $30^{\circ}$ irrespective of the cases in which the fibers are oriented in $x z$ - and $y z$-planes.

\section{Appendix}

In (6) and (7), the matrices $\left[Z_{1}\right],\left[Z_{2}\right],\left[Z_{3}\right],\left[Z_{4}\right],\left[Z_{5}\right]$, and $\left[Z_{6}\right]$ are given by

$$
\begin{gathered}
{\left[Z_{1}\right]=\left[\begin{array}{lll}
{\left[\bar{Z}_{1}\right]} & \tilde{0} & \tilde{0}
\end{array}\right],} \\
{\left[Z_{2}\right]=\left[\begin{array}{lll}
\left(\frac{h}{2}\right) I & {\left[\bar{Z}_{2}\right]} & \widetilde{0}
\end{array}\right],}
\end{gathered}
$$

$$
\begin{aligned}
& {\left[Z_{3}\right]=\left[\left(\frac{h}{2}\right) I \quad h_{v} I\left[\begin{array}{ll}
\left.\bar{Z}_{3}\right]
\end{array}\right]\right.} \\
& {\left[Z_{4}\right]=\left[\begin{array}{llllll}
\bar{I} & \overline{0} & \overline{0} & z \bar{I} & \overline{0} & \overline{0}
\end{array}\right],} \\
& {\left[Z_{5}\right]=\left[\begin{array}{llll}
\overline{0} & \bar{I} & \overline{0} & \left(\frac{h}{2}\right) \bar{I} \\
& \left(z-\frac{h}{2}\right) \bar{I} & \overline{0}
\end{array}\right],}
\end{aligned}
$$

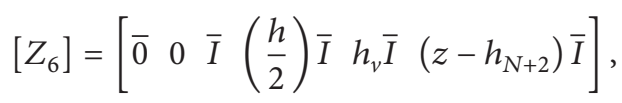

in which

$$
\begin{aligned}
& {\left[\bar{Z}_{1}\right]=\left[\begin{array}{cccc}
z & 0 & 0 & 0 \\
0 & z & 0 & 0 \\
0 & 0 & z & 0 \\
0 & 0 & 0 & 1
\end{array}\right]} \\
& {\left[\bar{Z}_{2}\right]=\left[\begin{array}{cccc}
\left(z-\frac{h}{2}\right) & 0 & 0 & 0 \\
0 & \left(z-\frac{h}{2}\right) & 0 & 0 \\
0 & 0 & \left(z-\frac{h}{2}\right) & 0 \\
0 & 0 & 0 & 1
\end{array}\right] \text {, }} \\
& {\left[\bar{Z}_{3}\right]=\left[\begin{array}{cccc}
\left(z-h_{N+2}\right) & 0 & 0 & 0 \\
0 & \left(z-h_{N+2}\right) & 0 & 0 \\
0 & 0 & \left(z-h_{N+2}\right) & 0 \\
0 & 0 & 0 & 1
\end{array}\right]} \\
& {[I]=\left[\begin{array}{llll}
1 & 0 & 0 & 0 \\
0 & 1 & 0 & 0 \\
0 & 0 & 1 & 0 \\
0 & 0 & 0 & 0
\end{array}\right], \quad[\bar{I}]=\left[\begin{array}{ll}
1 & 0 \\
0 & 1
\end{array}\right],} \\
& {[\overline{0}]=\left[\begin{array}{ll}
0 & 0 \\
0 & 0
\end{array}\right], \quad[\widetilde{0}]=\left[\begin{array}{cc}
\widetilde{0} & \tilde{0} \\
\widetilde{0} & \widetilde{0}
\end{array}\right] \text {. }}
\end{aligned}
$$

The various submatrices $B_{t b i}, B_{r b i}$, and $B_{r s i}$, appearing in (21), are given by

$$
B_{t b i}=\left[\begin{array}{ccc}
\frac{\partial \eta_{i}}{\partial x} & 0 & 0 \\
0 & \frac{\partial \eta_{i}}{\partial y} & 0 \\
\frac{\partial \eta_{i}}{\partial y} & \frac{\partial \eta_{i}}{\partial x} & 0 \\
0 & 0 & 0
\end{array}\right], \quad B_{t s i}=\left[\begin{array}{ccc}
0 & 0 & \frac{\partial \eta_{i}}{\partial x} \\
0 & 0 & \frac{\partial \eta_{i}}{\partial y}
\end{array}\right]
$$




$$
\begin{gathered}
\bar{B}_{r b i}=\left[\begin{array}{ccc}
\frac{\partial \eta_{i}}{\partial x} & 0 & 0 \\
0 & \frac{\partial \eta_{i}}{\partial y} & 0 \\
\frac{\partial \eta_{i}}{\partial y} & \frac{\partial \eta_{i}}{\partial x} & 0 \\
0 & 0 & 1
\end{array}\right], \quad B_{r b i}=\left[\begin{array}{ccc}
\bar{B}_{r b i} & \overline{0} & 0 \\
\hat{0} & \bar{B}_{r b i} & \overline{0} \\
\hat{0} & \overline{0} & \bar{B}_{r b i}
\end{array}\right], \\
B_{r s i}=\left[\begin{array}{ccc}
\bar{I} & \breve{0} & \breve{0} \\
\breve{0} & \bar{I} & \breve{0} \\
\breve{0} & \breve{0} & \bar{I}^{\prime} \\
\bar{B}_{r s i} & \breve{0} & \breve{0} \\
\breve{0} & \bar{B}_{r s i} & \breve{0} \\
\breve{0} & \breve{0} & \bar{B}_{r s i}
\end{array}\right], \quad \tilde{I}=\left[\begin{array}{lll}
1 & 0 & 0 \\
0 & 1 & 0
\end{array}\right],
\end{gathered}
$$

wherein $\widehat{0}$ and $\breve{0}$ are the $(3 \times 3)$ and $(2 \times 3)$ null matrices, respectively.

\section{References}

[1] T. Bailey and J. E. Hubbard, "Distributedpiezoelectric polymer active vibration control of a cantilever beam," Journal of Guidance, Control, and Dynamics, vol. 8, no. 5, pp. 605-611, 1985.

[2] E. F. Crawley and J. de Luis, "Use of piezoelectric actuators as elements of intelligent structures," AIAA Journal, vol. 25, no. 10, pp. 1373-1385, 1987.

[3] A. Baz and S. Poh, "Performance of an active control system with piezoelectric actuators," Journal of Sound and Vibration, vol. 126, no. 2, pp. 327-343, 1988.

[4] C. K. Lee, W. W. Chiang, and O. Sulivan, "Piezoelectric modal sensor/actuator pairs for critical active damping vibration control," Journal of the Acoustical Society of America, vol. 90, no. 1, pp. 374-384, 1991.

[5] S. Hanagud, M. W. Obal, and A. J. Calise, "Optimal vibration control by the use of piezoceramic sensors and actuators," Journal of Guidance, Control, and Dynamics, vol. 15, no. 5, pp. 1199-1206, 1992.

[6] Y. Gu, R. L. Clark, C. R. Fuller, and A. C. Zander, "Experiments on active control of plate vibration using piezoelectric actuators and polyvinylidene fluoride (PVDF) modal sensors," Journal of Vibration and Acoustics, Transactions of the ASME, vol. 116, no. 3, pp. 303-308, 1994.

[7] A. Baz and S. Poh, "Optimal vibration control with modal positive position feedback," Optimal Control Applications and Methods, vol. 17, no. 2, pp. 141-149, 1996.

[8] A. Kugi, K. Schlacher, and H. Irschik, "Infinite-dimensional control of nonlinear beam vibrations by piezoelectric actuator and sensor layers," Nonlinear Dynamics, vol. 19, no. 1, pp. 71-91, 1999.

[9] D. Sun, L. Tong, and D. Wang, "Vibration control of plates using discretely distributed piezoelectric quasi-modal actuators/sensors," AIAA Journal, vol. 39, no. 9, pp. 1766-1772, 2001.
[10] G. Caruso, S. Galeani, and L. Menini, "Active vibration control of an elastic plate using multiple piezoelectric sensors and actuators," Simulation Modelling Practice and Theory, vol. 11, no. 5-6, pp. 403-419, 2003.

[11] X. Zhang and A. G. Erdman, "Optimal placement of piezoelectric sensors and actuators for controlled flexible linkage mechanisms," Journal of Vibration and Acoustics, Transactions of the ASME, vol. 128, no. 2, pp. 256-260, 2006.

[12] G. Gatti, M. J. Brennan, and P. Gardonio, "Active damping of a beam using a physically collocated accelerometer and piezoelectric patch actuator," Journal of Sound and Vibration, vol. 303, no. 3-5, pp. 798-813, 2007.

[13] M. K. Kwak, S. Heo, and M. Jeong, "Dynamic modelling and active vibration controller design for a cylindrical shell equipped with piezoelectric sensors and actuators," Journal of Sound and Vibration, vol. 321, no. 3-5, pp. 510-524, 2009.

[14] I. Bruant, L. Gallimard, and S. Nikoukar, "Optimal piezoelectric actuator and sensor location for active vibration control, using genetic algorithm," Journal of Sound and Vibration, vol. 329, no. 10, pp. 1615-1635, 2010.

[15] Y. Fu, J. Wang, and Y. Mao, "Nonlinear vibration and active control of functionally graded beams with piezoelectric sensors and actuators," Journal of Intelligent Material Systems and Structures, vol. 22, no. 18, pp. 2093-2102, 2011.

[16] F. Chen, M. Hong, M. Song, and H. Cui, "Optimal control of a beam with discontinuously distributed piezoelectric sensors and actuators," Journal of Marine Science and Application, vol. 11, no. 1, pp. 44-51, 2012.

[17] B. Azvine, G. R. Tomlinson, and R. J. Wynne, "Use of active constrained-layer damping for controlling resonant vibration," Smart Materials and Structures, vol. 4, no. 1, pp. 1-6, 1995.

[18] A. Baz and J. Ro, "Vibration control of plates with active constrained layer damping," Smart Materials and Structures, vol. 5, no. 3, pp. 272-280, 1996.

[19] C. Chantalakhana and R. Stanway, "Active constrained layer damping of clamped-clamped plate vibrations," Journal of Sound and Vibration, vol. 241, no. 5, pp. 755-777, 2001.

[20] J. Ro and A. Baz, "Optimum placement and control of active constrained layer damping using modal strain energy approach," JVC/Journal of Vibration and Control, vol. 8, no. 6, pp. 861-876, 2002.

[21] M. C. Ray and J. N. Reddy, "Optimal control of thin circular cylindrical laminated composite shells using active constrained layer damping treatment," Smart Materials and Structures, vol. 13, no. 1, pp. 64-72, 2004.

[22] C. M. A. Vasques, B. R. Mace, P. Gardonio, and J. D. Rodrigues, "Arbitrary active constrained layer damping treatments on beams: finite element modelling and experimental validation," Computers and Structures, vol. 84, no. 22-23, pp. 1384-1401, 2006.

[23] F. M. Li, K. Kishimoto, K. Wang, Z. B. Chen, and W. H. Huang, "Vibration control of beams with active constrained layer damping," Smart Materials and Structures, vol. 17, no. 6, Article ID 065036, 2008.

[24] L. Yuan, Y. Xiang, Y. Huang, and J. Lu, "A semi-analytical method and the circumferential dominant modal control of circular cylindrical shells with active constrained layer damping treatment," Smart Materials and Structures, vol. 19, no. 2, Article ID 025010, 2010.

[25] N. Kumar and S. P. Singh, "Vibration control of curved panel using smart damping," Mechanical Systems and Signal Processing, vol. 30, pp. 232-247, 2012. 
[26] W. A. Smith and B. A. Auld, "Modeling 1-3 composite piezoelectrics: thickness-mode oscillations," IEEE Transactions on Ultrasonics, Ferroelectrics, and Frequency Control, vol. 38, no. 1, pp. 40-47, 1991.

[27] Piezocomposites, Material Systems Inc., 543 Great Road, Littleton, MA, 01460.

[28] Y. Benveniste and G. J. Dvorak, "Uniform fields and universal relations in piezoelectric composites," Journal of the Mechanics and Physics of Solids, vol. 40, no. 6, pp. 1295-1312, 1992.

[29] M. L. Dunn and M. Taya, "Micromechanics predictions of the effective electroelastic moduli of piezoelectric composites," International Journal of Solids and Structures, vol. 30, no. 2, pp. 161-175, 1993.

[30] J. Aboudi, "Micromechanical prediction of the effective coefficients of thermo-piezoelectric multiphase composites," Journal of Intelligent Material Systems and Structures, vol. 9, no. 9, pp. 713-722, 1999.

[31] X. Ruan, T. W. Chou, A. Safari, and S. C. Danforth, "A 3-D connectivity model for effective piezoelectric properties of yarn composites," Journal of Composite Materials, vol. 36, no. 14, pp. 1693-1708, 2002.

[32] A. Baz and A. Tempia, "Active piezoelectric damping composites," Sensors and Actuators A, vol. 112, no. 2-3, pp. 340-350, 2004.

[33] H. Berger, S. Kari, U. Gabbert et al., "Unit cell models of piezoelectric fiber composites for numerical and analytical calculation of effective properties," Smart Materials and Structures, vol. 15, no. 2, pp. 451-458, 2006.

[34] M. Sakthivel and A. Arockiarajan, "An analytical model for predicting thermo-electro-mechanical response of 1-3 piezoelectric composites," Computational Materials Science, vol. 48, no. 4, pp. 759-767, 2010.

[35] M. C. Ray and A. K. Pradhan, "On the use of vertically reinforced 1-3 piezoelectric composites for hybrid damping of laminated composite plates," Mechanics of Advanced Materials and Structures, vol. 14, no. 4, pp. 245-261, 2007.

[36] M. C. Ray and A. K. Pradhan, "Performance of vertically and obliquely reinforced 1-3 piezoelectric composites for active damping of laminated composite shells," Journal of Sound and Vibration, vol. 315, no. 4-5, pp. 816-835, 2008.

[37] S. K. Sarangi and M. C. Ray, "Smart damping of geometrically nonlinear vibrations of laminated composite beams using vertically reinforced 1-3 piezoelectric composites," Smart Materials and Structures, vol. 19, no. 7, Article ID 075020, 2010.

[38] A. Krishnan and J. V. Deshpande, "Vibration of skew laminates," Journal of Sound and Vibration, vol. 153, no. 2, pp. 351-358, 1992.

[39] A. R. Krishna Reddy and R. Palaninathan, "Free vibration of skew laminates," Computers and Structures, vol. 70, no. 4, pp. 415-423, 1999.

[40] C. S. Babu and T. Kant, "Two shear deformable finite element models for buckling analysis of skew fibre-reinforced composite and sandwich panels," Composite Structures, vol. 46, no. 2, pp. 115-124, 1999.

[41] A. K. Garg, R. K. Khare, and T. Kant, "Free vibration of skew fiber-reinforced composite and sandwich laminates using a shear deformable finite element model," Journal of Sandwich Structures and Materials, vol. 8, no. 1, pp. 33-53, 2006.

[42] J. N. Reddy, Mechanics of Laminated Composites Plates Theory and Analysis, CRC press, Boca Raton, Fla, USA, 1996. 

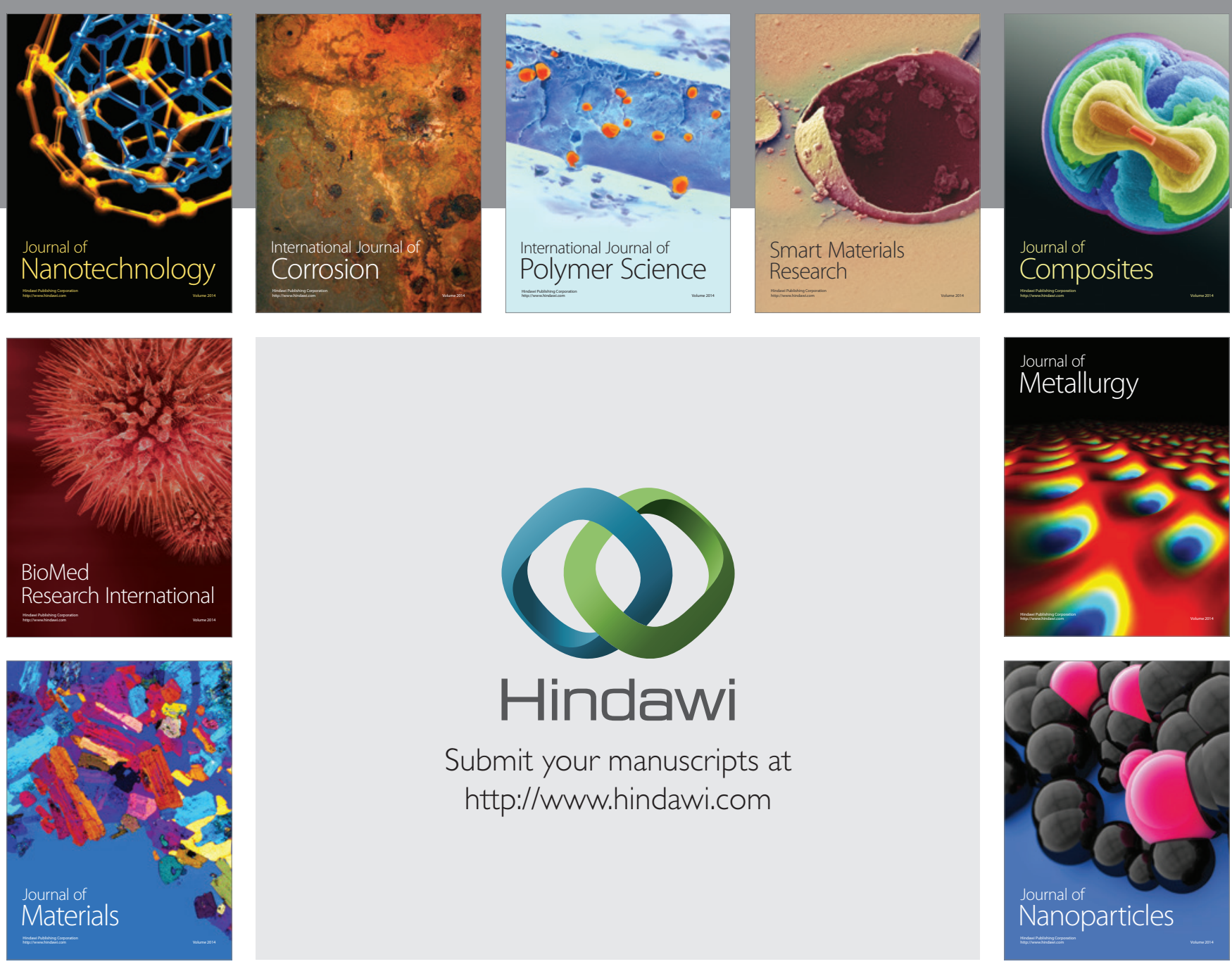

Submit your manuscripts at http://www.hindawi.com
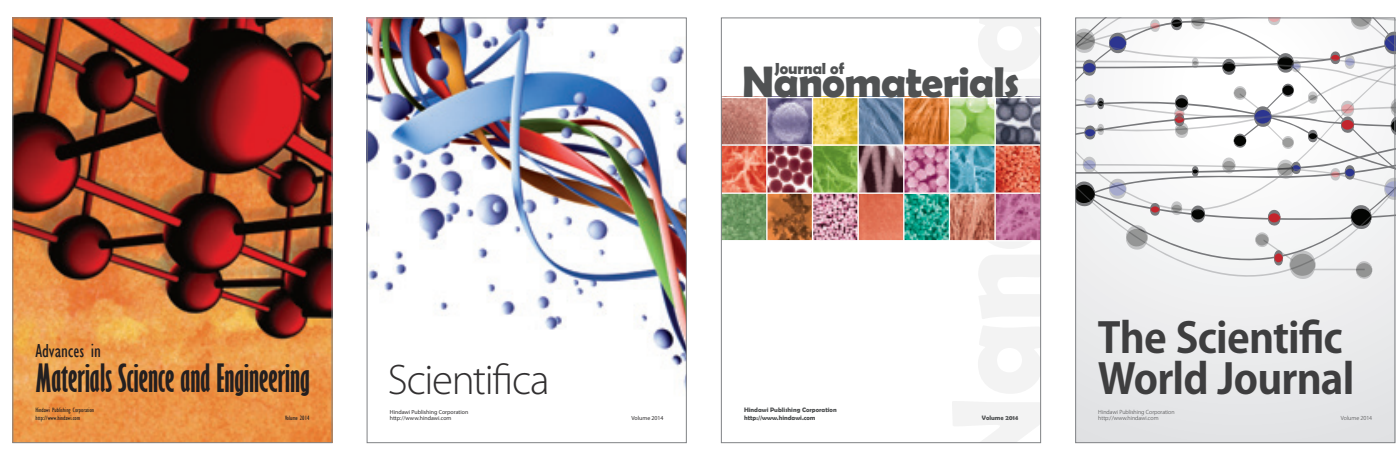

\section{The Scientific World Journal}
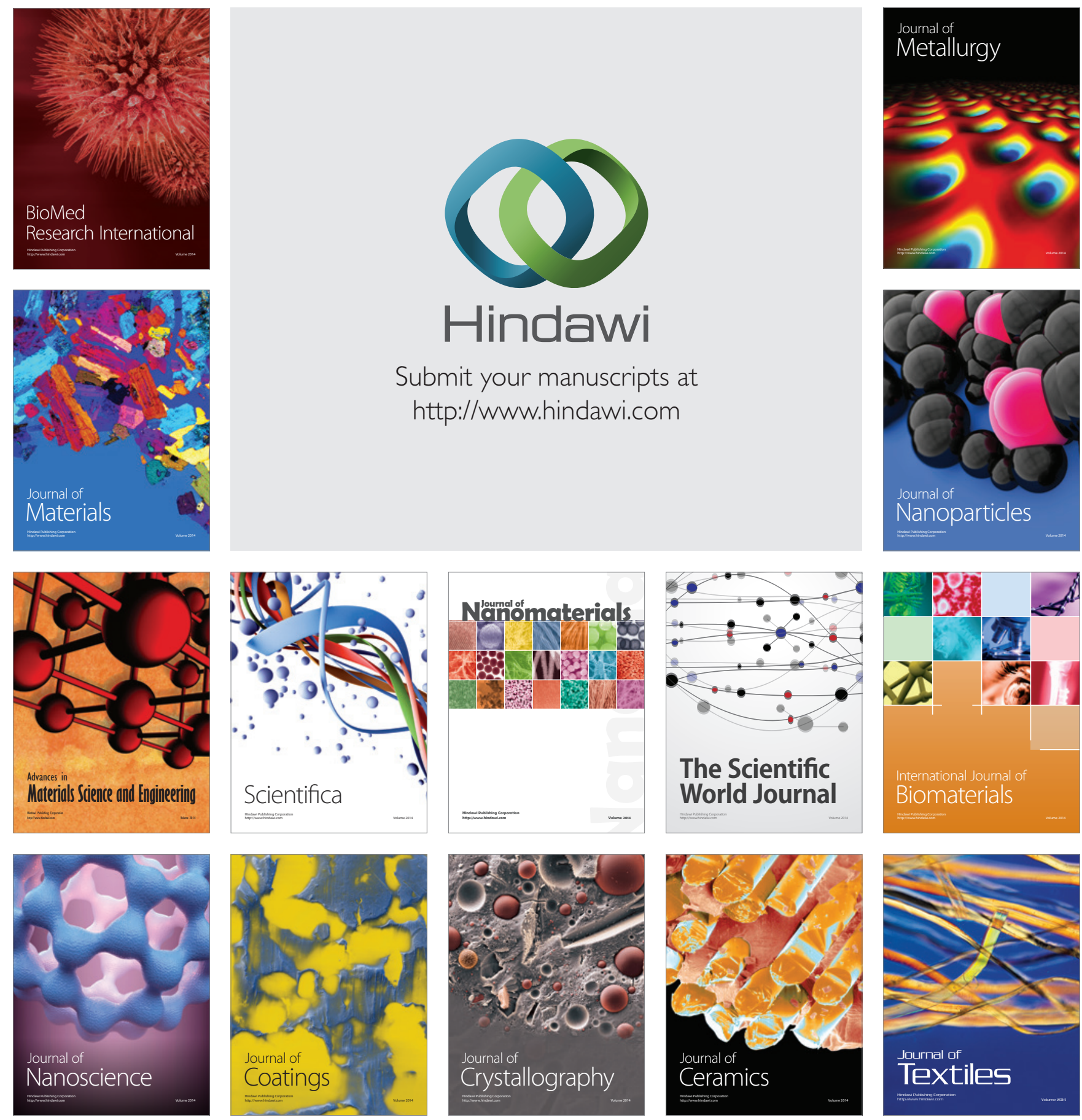